\title{
On Forecasting Lung Cancer Patients' Survival Rates Using 3D Feature Engineering
}

\author{
By \\ Tahira Ghani \\ A thesis proposal submitted to \\ the Faculty of Graduate and Postdoctoral Affairs \\ in partial fulfilment of \\ the requirements for the degree of \\ Master of Computer Science \\ Ottawa-Carleton Institute for Computer Science \\ School of Computer Science \\ Carleton University \\ Ottawa, Ontario
}

September 2019

(C) Copyright

2019, Tahira Ghani 
The undersigned hereby recommend to the Faculty of Graduate and Postdoctoral Affairs acceptance of the thesis,

\title{
On Forecasting Lung Cancer Patients' Survival Rates
}

\section{Using 3D Feature Engineering}

submitted by

\section{Tahira Ghani}

\author{
Dr. Michel Barbeau \\ (Director, School of Computer Science) \\ Dr. B. John Oommen \\ (Thesis Supervisor)
}

Carleton University

September 2019 


\section{Abstract}

This thesis focuses on the application of Machine Learning in the healthcare domain, by extracting meaningful information from medical image reports using image processing techniques. Rather than considering a quintessential classification problem, however, we consider a regression problem whereby we aim to predict the survival times of patients after they have been diagnosed with adenocarcinoma, a type of lung cancer. We attempt to achieve this by processing chest CT scans to isolate the cancerous nodules, and extracting relevant features from the images of the nodules.

Our work proposes the engineering of a feature set that can qualify the tumours with traits that are not visible to the naked eye using textural and statistical measures. We first consider the data in its 2D form, i.e., pixel data directly obtained from the images themselves, and create a benchmark using 2D Haralick computations. This feature set is then appended with statistical shape measurements as well.

Furthermore, we offer two additional schemes in feature set generation, the first acting as a causal to the second. Taking the 2D feature set, we analyze the feature measurements in relation to the tumour depth as the tumour progresses through the slices of the scan. Cumulating this knowledge into a single measurement not only significantly improves the regression results, but also demonstrates the advantage of focusing on the prediction of short-term survival rate timelines as opposed to longterm survival rate timelines.

The second scheme, founded by the results obtained by the first, concentrates on considering the cancer nodule in its 3D entirety, rather than just the images. This results in a considerably large feature space, with over 100 dimensions. To process these, we explore dimensionality reduction techniques, particularly data diagonalization in a block-diagonal matrix manner, to further enhance regression results. 


\section{Acknowledgements}

First and foremost, I would like to acknowledge my Supervisor Prof. B. John Oommen, for his utmost support and guidance throughout the journey of this thesis. He has been readily available for any type of discussion, whether that required phone meetings due to my work shifts, or last minute revision meetings before his flights amidst his busy travel schedule. His door was always open. His knowledge in the domain of pattern recognition is profound and has been vital in the completion of this research.

I would like to offer a huge thanks to the clinical collaborators from the Ottawa Heart Institute, Dr. Joao Inacio and Dr. Rebecca Thornhill, for their time, effort, direction and expertise in the medical domain. Without them, this thesis would not have been possible.

Additionally, a special thanks to my mentor at work, John Howat, for dedicating time to proofread and provide objective feedback for the content of this thesis, as well as for answering my never-ending questions with unwavering patience.

Finally, I would like to sincerely thank my support circle starting with my parents, for encouraging me in all the endeavours I pursue, especially this program and research. I thank my siblings, and specifically my brother, Mohammad Ghani, who has been my biggest advocate. I am also grateful to my dear friend, Sajeda Almalki, who has given me many motivational "pep" talks and constantly checked in on my progress. Besides them, I am grateful to the numerous friends whom I cannot mention here, due to the space constraints.

I am immensely grateful to the School of Computer Science, the faculty and the staff as well as the friends I have made here for making this journey pleasant and fulfilling. 


\section{Contents}

1 Introduction 1

1.1 Introduction . . . . . . . . . . . . . . . . . . . 1

1.2 Motivation of the Thesis . . . . . . . . . . . . . . . 3

1.3 Objectives of the Thesis . . . . . . . . . . . . . . . . . 4

1.3.1 Data Source . . . . . . . . . . . . . . . 5

1.3.2 Problem Statement . . . . . . . . . . . . . . . 5

1.4 Contributions of the Thesis . . . . . . . . . . . . . 6

1.5 Organization of the Thesis . . . . . . . . . . . . . . 7

2 Survey of the Field $\quad 9$

2.1 Introduction . . . . . . . . . . . . . . . . . . . . . 9

2.2 Computer Science Aspects . . . . . . . . . . . . . . . . . . . . . . . . 10

2.2.1 Machine Learning for Images . . . . . . . . . . . . . . . . . . 10

2.3 Medical Aspects . . . . . . . . . . . . . . . . . . . . . . 17

2.3.1 Computed Tomography Scans . . . . . . . . . . . . . . . . . . 18

2.3.2 Lung Abnormalities in Chest CT Scans . . . . . . . . . . . . . 21

2.4 Computer Science in Medical Imaging . . . . . . . . . . . . . . . . . . 22

2.4.1 Chest CT Scan Preprocessing . . . . . . . . . . . . . . . 22

2.4.2 Lung Segmentation . . . . . . . . . . . . . . . . . . . . 23

2.4.3 Nodule Detection and Segmentation . . . . . . . . . . . . 25

2.4.4 Nodule and Texture Feature Extraction . . . . . . . . . . . . . 30

2.5 Chapter Conclusions . . . . . . . . . . . . . . . . . . . . . 33 
3 Lung Abnormality Detection 34

3.1 Introduction . . . . . . . . . . . . . . . . . 34

3.2 Foundational Algorithm . . . . . . . . . . . . . . 34

3.2.1 Preprocessing . . . . . . . . . . . . . . 37

3.2.2 Lung Segmentation . . . . . . . . . . . . . . 38

3.2.3 Feature Extraction ................ . . 40

3.2.4 Texture Analysis . . . . . . . . . . . . . . . . . 41

3.2.5 Results...................... 44

3.3 Additional Work . . . . . . . . . . . . . . . 47

3.3.1 Lung Tumour Segmentation . . . . . . . . . . . . 47

3.3.2 3D Nodule Construction . . . . . . . . . . . . . . . 48

3.4 Chapter Conclusions . . . . . . . . . . . . . . . . . . . 48

4 Enhanced Regression: 2D to 3D Features 50

4.1 Introduction . . . . . . . . . . . . . . . . . 50

4.2 Generating a Benchmark . . . . . . . . . . . . . . 51

4.2.1 Model Evaluation . . . . . . . . . . . . . . . . 51

4.2 .2 Baseline Results . . . . . . . . . . . . . . . . . . 52

4.3 Benchmark Feature Set Modification . . . . . . . . . . . . 54

4.3.1 Shape Feature Calculation . . . . . . . . . . . . . . 54

4.3.2 Shape-Based Feature Analysis . . . . . . . . . . . . . 56

4.4 Chapter Conclusions . . . . . . . . . . . . . . . . . 60

$5 \quad 3 D$ Features Exploration and Analysis $\quad 63$

5.1 Introduction . . . . . . . . . . . . . . . 63

5.2 3D Feature Set Compilation . . . . . . . . . . . . . 64

5.2.1 Evaluation of Feature Space . . . . . . . . . . . . . 67

5.3 Feature Selection Techniques . . . . . . . . . . . . . 67

5.3.1 Short-term Prediction Results . . . . . . . . . . . . . . 69

5.4 Dimensionality Reduction Techniques . . . . . . . . . . . . 69

5.4.1 Principal Component Analysis (PCA) . . . . . . . . . . . 70

5.5 Data Diagonalization . . . . . . . . . . . . . . 71 
5.5.1 Implementation and Results . . . . . . . . . . . . . . 73

5.6 Chapter Conclusions . . . . . . . . . . . . . . . 74

6 Conclusion $\quad 76$

6.1 Summary ........................... 76

6.2 Conclusion and Achievements . . . . . . . . . . . . . 78

6.3 Future Work . . . . . . . . . . . . . . . . 79

$\begin{array}{ll}\text { Appendices } & 86\end{array}$

A Appendix A - Haralick Features and Formulae $\quad 87$ 


\section{List of Figures}

2.1 Planes captured in a computed tomography scan. . . . . . . . . . . 19

2.2 Window adjustments in Chest CT scans, sourced from RadiANT DICOM Viewer Manual. . . . . . . . . . . . . . . . . . . . . . . 21

2.3 Dense structures attached to lung walls. . . . . . . . . . . . . . 24

2.4 The Central focused CNN structure, including convolutional layers, central pooling and connection layer (sourced from [41]). . . . . . . 29

3.1 Process flow of the prior abnormality detection algorithm. . . . . . 36

3.2 Different Chest CT views based on window parameter adjustments. . 38

3.3 ROI extraction. . . . . . . . . . . . . . . . . . . . . . . . 39

3.4 Lung extraction. . . . . . . . . . . . . . . . . . . . . . . . 39

3.5 Detection of abnormal air and dense consolidation regions. . . . . . 42

3.6 "Abnormal" texture patches. . . . . . . . . . . . . . . . 44

3.7 "Normal" texture patches. . . . . . . . . . . . . . . . . 45

3.8 Highlighted abnormally classified texture patches. . . . . . . . . 46

3.93 3D visualization of tumour nodule, sourced from [39] . . . . . . . . 48

4.1 Correlation heatmap of the 2D Benchmark feature set. . . . . . . 54

4.2 Tumour shape feature calculation process. . . . . . . . . . . 55

4.3 A visualization of masks of tumours from 2D images as the nodule progresses throughout the thoracic region. . . . . . . . . . . 57 
4.4 Plots of the Mean of the Area based on the tumour's progression for binned survival times between 0 months and 36 months. . . . . . . . 58

4.4 Plots of the Mean of the Area based on the tumour's progression for binned survival times between 36 months and 60 months. . . . . . . . 59

5.1 A visualization of PCA. . . . . . . . . . . . . . 70

5.2 Block diagonal matrix. . . . . . . . . . . . . . . . . . . . . . . 72 


\section{1 \\ Introduction}

\section{$1.1 \quad$ Introduction}

Artificial Intelligence (AI), with its goal to mimic human cognitive abilities, is a highly technical field in terms of theoretical research, but has expanded into practical applications in a variety of domains. The development of the AI ideology dates back to the 1950's, with notable figures such as Claude Shannon (known as the "father of information theory") publishing the first article on the development of a chess-playing computer program, as well as Alan Turing and his famous Turing Test.

The exponential increase in data over the past few decades, however, has been a pivotal energy in the field of AI. Machine learning (ML), a subset of AI, has leveraged the copious amounts of data being generated at an ever increasing rate. Perhaps it could even be said that the conception and rapid advancement of ML is due to the 
rate at which data has grown. While the aim of AI is broad in nature, i.e., to simulate human intelligence with any form of smart decision making, ML algorithms additionally aim to perform learning on any given data to predict future characteristics, depending on the context at hand. This behaviour and the promise of potential has been displayed in every possible adjunct field. ML has expanded in at least two specific umbrellas which have profoundly impacted the common man's daily life. Both of these are based on the concept of pattern recognition, explained below:

1. Computer Vision (CV) - refers to the field where computers attempt to understand and process the content of digital images. CV encompasses a significant portion of image processing (IP), but is notably distinct. CV aims to extract useful information from images, such as object detection and classification, rather than simply perform operations on the data. This has since conceptualized into many practical applications including, but not limited to, automotive safety (with realtime object detection on video footage from cameras on cars), fingerprint and facial recognition, medical imaging, and automated checkouts.

2. Natural Language Processing (NLP) - refers to the field where computers attempt to understand, analyze, manipulate and reproduce human language, both, written and verbal. NLP has resulted in applications involving speech recognition for assistive technology (Siri, Alexa, Google Home), spam filters, tools for sentiment analysis, auto-correction and prediction, among many others.

The applications of ML and computer vision in the healthcare domain, in particular, have led to significant interest from research communities all over the world due to its numerous implications. A number of notable research journals have emerged specifically focusing on the interaction of these two fields, such as Artificial Intelligence in Medicine, Computerized Medical Imaging and Graphics, Medical Image Analysis, Computer Methods and Programs in Biomedicine, and Biomedical Signal Processing and Control, to name a few. The palpable synergy between the two spheres, both supported by sufficient research, can revolutionize modern healthcare. 


\subsection{Motivation of the Thesis}

Advancements in technology have managed to replace manpower in many fields. For healthcare, this began in the 1960's, with attempts to automate the systematic diagnosis of pathologies in the medical field. The goal of these endeavours was to replace healthcare professionals. Two decades later, in the 1980's, however, the concept of automated computer diagnosis changed to Computer-Aided Diagnosis (CAD) due to the complex nature of healthcare and medicine. This progression of thought came about when researchers began to realize that while computerized decision-making for severe illnesses would encompass an extremely high risk factor, radiologists could, at the very least, utilize the output of assistive computer programs [16]. CAD-based research ranges over multiple forms of radiology and focuses on numerous organs such as the brain, chest (lung, heart, etc.), liver, skeletal system, among many others.

The first wave of clinical computer vision applications revolved around image processing and image reconstruction. These pieces of software were readily accepted for practical use in medical offices and hospitals, as they are easily perceived as an assistive tool for specialists involved in interpreting and reading scans. Examples of such software include user-led segmentation of regions of interest, quantitative measurements, and 3D reconstructions of anatomical structures (vessels, arteries, nodules, organs, etc.). This functionality enabled healthcare specialists to better analyze medical scans and reports, and with extensive development has also resulted in aiding surgical planning. However, it did not provide a predictive analysis or second opinion for the patient under consideration.

Research for computer vision in medicine advanced to focus on automatic segmentations, feature extraction and classification for the presence of specific diseases or pathologies. CAD systems are divided into two sub-categories, computer-aided detection (CADe) and computer-aided diagnosis (CADx) [46]. Both branches are being actively researched, with CADe being more focused on computationally-efficient early detection with a higher sensitivity and low false-positive rate, and CADx being more focused on the further characterization and classification of such lesions.

The most famous use-case for such an application is the detection (or classification) 
of a nodule being cancerous. However, if we can push this one step further and are able to judge the severity of a cancerous nodule, the prognosis and determination of treatment plans can be adjusted to yield a greater chance of success. Such an analysis would include qualifying tumours in manners that are not visible to the naked eye, by invoking statistical and textural measures. With sufficient research, the practical application of such a problem could be a breakthrough in the healthcare process.

\subsection{Objectives of the Thesis}

The main objective of this thesis is to explore and investigate the existence of relations between statistical measures with regards to the texture and shape of a nodule classified as adenocarcinoma (a type of cancer) to the survival time of the patient post-diagnosis. It is important to note that the task at hand is a regression problem, instead of the more commonplace classification problem traditionally considered in healthcare.

When considering the applications of ML in healthcare, classification problems have been the dominant area of focus such that the presence, or lack thereof, of a specified anatomical structure can be stated. However, transforming the context of the application to a regression domain can enable a critical advancement in CAD systems. By suggesting a survival time for a given patient, the trajectory of the illness and treatment plan can be evaluated at a deeper level. Through the literature review, discussed in more detail in Chapter 2, we have identified a significant gap in the presence of regression analyses for pathologies.

We aim to engineer a prognostic feature set based on quantitative measurements that are not visible with a simple glance or reading of the scan, and aspire to reduce the inherent subjectivity and variability when evaluating medical reports with such measures.

We adapt the research goal of Grove et al. [20] by computing and analyzing features that are indicative of the severity of the cancerous nodule, whereby severity can be considered as synonymous with the survival time of a patient after the diagnosis of the cancer. Whereas their study was, for the most part, a hypothesis-based testing 
methodology, ours will be more explorative in nature with the goal of finding descriptive quantitative measurements. We will divide the feature extraction into two main approaches, the first involving 2D images, and the second considering the nodule in its 3D entirety.

\subsubsection{Data Source}

In this thesis, we will use the publicly available data from The Cancer Imaging Archive $^{1}$ (TCIA), a service which hosts an archive of data for de-identified medical images of cancer. The dataset used for this thesis is the "LungCT-Diagnosis" data [20] on TCIA, uploaded in 2014. The set consists of CT scans for 61 patients that have been diagnosed with adenocarcinoma, a type of lung cancer, with the number of images totalling up to about 4,600 over all the scans. However, considering only images that have the presence of a cancer nodule, the count reduces to approximately 450 images. Understandably, since we take the entire scan in consideration for the 3D aspect, 61 data points is on the smaller size for a ML dataset, but we are, of course, constrained to work with what we have. As we will see, it suffices for the purpose of regression analyses. The dataset also includes the clinical metadata, where the survival time of the patient associated with each scan, is listed. The CT scans were reviewed, and the cancer tumours were manually segmented with the validation of a clinical doctor from the Ottawa Heart Institute, to whom we are extremely grateful.

\subsubsection{Problem Statement}

For the sake of clarity, we concisely specify our problem statement as the following:

- Transform the foundational problem domain to concentrate on a more narrowed focus on the cancer nodules themselves, rather than the entire lung, as well as shifting the context from classification to a regression analysis.

- Engineer the descriptive feature sets through exploration of statistical texture and shape measures in both 2D and 3D feature spaces.

\footnotetext{
${ }^{1}$ More information can be found at https://www.cancerimagingarchive.net/.
} 
- Analyze feature sets with data reduction and feature elimination techniques, to obtain the most succinct, but informative, collections of features.

\subsection{Contributions of the Thesis}

The contributions of this thesis are as follows:

- We have been able to demonstrate that there is a distribution for the shapes of the scans as they are processed sequentially along the depth of the tumour. From this sequence of images, we have been able to obtain relatively simple indices that relate to some geometric features of the sequence, and that possess phenomena applicable to classification and regression.

- Using the sequential processing of images, we enhanced the regression results by almost $30 \%$ on the entire dataset, and achieved a relative error of as low as $9 \%$ on the subset of data with a survival rate of 24 months or less. In this manner, we displayed that a short term prediction is more accurate and reliable.

- We were able to analyze and reduce the computational space of the 3D feature set through the implementation of multiple techniques. Notably, we were also able to simplify the data diagonalization technique through an ensemble methodology by processing the covariance matrix in a block-diagonal manner.

- The work done in this thesis has led to two research papers:

(a) The first, a submission to the Australian Joint Conference on Artificial Intelligence, where we have proved a strong correlation between shape features from the $2 \mathrm{D}$ images at successive layers of the scan and the survival rate of the patient.

(b) The second, yet to be submitted, will focus on the enhanced results achieved from dimensionality reduction through simplification of computational com-

plexity in the data diagonalization process, with the implementation of block-diagonalization. 


\subsection{Organization of the Thesis}

Chapter 2 presents an overview of the published literature in the fields of Image Processing (IP), medical imaging, and applications of both IP and ML in healthcare and diagnostics. We first discuss the applications of ML in IP, and the process of feature extraction and classification in the context of images. The chapter then examines the attributes of medical imaging, and more specifically, the images derived from Computed Tomography (CT) radiology. Lastly, but perhaps the most critical section in this chapter, is the survey of the literature regarding the application of IP in medical imaging such as the preprocessing techniques, Region of Interest (ROI) segmentation, nodule detection and classification, among many others.

Chapter 3 discusses the foundational algorithm used as the basis of this thesis. This algorithm was a previous work of the author done as part of a capstone project for a previous degree. The chapter discusses the objective and process flow of the CAD prototype, the IP techniques implemented for processing chest CT scans, as well as the various features extracted. We also briefly explain the performance of ML models for the purpose of a binary classification.

Chapter 4 marks the beginning of the novel work which this thesis focuses on. It considers the performance of regression models on the pre-computed texture-based feature set for the purpose of predicting survival times of patients diagnosed with adenocarcinoma as a benchmark for comparison. The feature set is analyzed and further appended with shape-based features. This chapter considers observations in the $2 \mathrm{D}$ domain, i.e., just images in the scan and not the entire scan itself, and presents the results of using the modified feature sets. However, the feature quantities are also compared against tumour-depth, and with relations between successive images of the scans in a $3 \mathrm{D}$ manner.

Chapter 5 focuses on the engineering of a 3D feature set, with the justifications of this exploration stated in the previous chapter. Quantitative measurements such as texture analysis are performed in an extended 3-dimensional manner through the 
implementation of the external framework, Pyradiomics. Due to the resulting highdimensional feature set, the remainder of the chapter focuses on feature selection and dimensionality reduction techniques with the aim to minimize error from the same regression models tested earlier.

Chapter 6 concludes the thesis. It discusses the results found in this thesis compared to results of research with a similar problem statement at hand. It also includes a summary of each chapter, and discusses avenues for future work. 


\section{2 Survey of the Field}

\section{$2.1 \quad$ Introduction}

This chapter ${ }^{1}$ will be a background survey of the literature in the medical image processing domain. We will first discuss Machine Learning (ML) concepts pertaining to Image Processing (IP), such as feature extraction, Pattern Recognition (PR), and the classification of images.

Thereafter, we will explore the medical domain. Biological imaging and radiology includes a variety of images such as X-rays, Magnetic Resonance Imaging (MRI), ultrasounds, tomography scans, etc. For the purpose of this thesis, our specific focus will lie in Computed Tomography (CT) imaging. We will discuss the different views present in the scan, cancer and interstitial disease statistics, and abnormalities in

\footnotetext{
${ }^{1}$ There are literally tens of thousands of papers and publications for the material presented in this chapter. The references that we cite are not necessarily the most important ones. Rather, they are representative of what the topic at hand covers.
} 
terms of the types of lung nodules and/or textures.

Finally, we will review the existing literature on the application of IP and PR on medical image reports. The work in this domain ranges through all the varieties of biological imaging and radiology listed above, with specific pathological goals at hand. We will consider which IP techniques have been applied for various reports, and which of these techniques have been successful in attaining consistent results.

\subsection{Computer Science Aspects}

An image is a visual representation of information, whether it be artificially generated or captured through a device. To machines, however, this information is communicated through bits and numbers called "pixels". IP techniques essentially deal with the manipulation of these pixels. We will consider these techniques in detail, with a brief discussion on the foundational theory, its usage and applications, as well as recording any benefits or drawbacks.

Additionally, we will do a field study on ML in the context of IP. This will include a focus on feature extraction and how IP techniques can aid in obtaining condensed quantitative measures possessing strong predictive capabilities for PR and image classification.

\subsubsection{Machine Learning for Images}

Machine learning, a prominent research domain, has gained significant traction in the past few decades. From researching the creation of algorithms based on mathematical models that learn the relations and predictive capabilities of features (i.e., quantitative measures), the field has progressed to real-time applications in widespread global industries, including finance, civil infrastructure, security, medicine and healthcare. Data scientists have "struck gold" with the rapid increase in digital data over the past decade, especially in unstructured formats, such as images and video.

One of the most famous applications of ML in image processing is in facial recognition. This has become a critical software in central surveillance and intelligence 
agencies. The domain has grown, however, to include more dynamic predictions and analysis such as detecting changes or emotional states from facial expressions, which can lead to more holistic applications such as profiling individuals through video footage of behaviour observation.

Another widely-known application of ML in image processing is character recognition. This branch of PR has been extended to more complex systems, such as document verification or the development of electronic libraries and multimedia databases, to name a few [26]. Due to the evolution of work in this domain, PR has become less expensive and more feasibly applicable in real-time applications.

Additionally, one of the most widely-used applications of computer vision is image retrieval. This application is relevant in many domains, and is also utilized by the common man in his or her daily life. It pertains to the system involved in browsing, searching and retrieving images from a large image database. There are, primarily, two techniques used for achieving this: Image meta search, where the search queries are created using the metadata such as captions or keywords, and Content-based Image Retrieval (CBIR), where CV techniques are employed in order to locate images that match the "query" based on similar textures, shapes, colors, etc.

\section{Feature Extraction}

Perhaps the most important part of ML applications with images and videos is feature extraction. The goal of feature extraction is to retrieve meaningful information, or a more concise representation of information, from the usually large and redundant input data. This set of quantitative measures is known as features, or a feature vector. It is desirable for these measures to possess high predictive capability and encompass the knowledge required to distinguish between output classes.

Feature extraction is applied to images after the pre-processing phase, which includes a combination of basic IP operations - varied depending on the image context at hand. Feature extraction is, generally, followed by feature selection, where features with a stronger relevance to the output class should not be removed from the feature set without accepting a loss in classification accuracy.

Image features can be categorized in two ways [12]: 
1. General versus domain-specific features - where these general features are independent of the application context (colour, shape, texture), and domain-specific features are conceptual for the context at hand.

2. Low-level versus high-level features - where high-level features are more complex and based on the low-level features extracted (eyes for facial recognition, fingerprints, lines for character recognition, etc.).

Furthermore, there is a notable difference between object detection and recognition. Detection of objects in an image focuses on global features which can describe the image as a whole. On the other hand, recognition is more of a classification problem, utilizing local processing of interest points and the description of local regions as features. Applications which fundamentally use interest points as features in images include image alignment (forming panoramas out of multiple images by detecting the same interest points in different images), image matching, 3D reconstruction, motion tracking, object recognition, and robot navigation.

Local features describe patches in images and detect areas of interest in an image as well as form a description of the region. Due to the focus on a small area, local features aim to achieve invariance in geometric (i.e., translational, rotational, and scaling) and photometric (i.e., brightness and exposure) aspects. Consequently, interest points can be correlated in different images regardless of the angle or lighting present. Local features have many advantages such as:

- Locality: indifferent to obstruction and noise

- Quantity: number in hundreds or thousands in a single image

- Distinctiveness: strong differentiating capabilities

- Efficiency: achieves good real-time performance.

Images are searched for interest points to choose positions for features. Interest points include a window of pixels where there is a lot of change and hence, involves extensive use of corner and edge detection techniques. 
A famous local feature extraction technique is the Scale Invariant Feature Transform (SIFT) descriptor, published by Lowe [28] in 1999. The SIFT feature vector is computed by first detecting the key pixels, selected from the local minima and maxima using the difference of the Gaussian function. It then calculates the gradient around a key pixel based on a window size, followed by applying quadrants and bins to form an 8-orientation histogram bin.

Since the introduction of the SIFT technique, there has been much work aiming to improve the derived feature vector. Ke and Sukthankar [24] suggested normalizing the gradient patch through application of Principal Component Analysis (PCA). They achieved descriptors that proved to be more unique, robust, and compact than the traditional SIFT descriptor on datasets that were intentionally distorted, as well as on abstract images such as graffiti. Mortensen et al. [30] proposed a modification to the SIFT feature by enhancing the descriptor with a global context vector to expand the neighbourhood of interest. Cheung and Hamarneh [11] introduce the n-dimensional SIFT vector to accommodate multidimensional images by hyperspherical coordinates for gradients and multidimensional histograms.

Inspired by SIFT, is another extensively-applied approach known as the Speed Up Robust Feature (SURF) extraction. Bay et al. [6] approximated the LoG with box filters, as opposed to the difference of Gaussian used in SIFT. Box filters enable convolution and parallelism in the computation, achieving faster speed.

Analyzing textures in an image is also an important characteristic when it comes to feature extraction. One of the earliest proposed techniques, still widespread in IP applications today, was the Grey-Tone Spatial-Dependence Matrices (GTSDM), published by Haralick et al. [22]. This is more-commonly known as the Grey-Level Co-occurrence Matrices (GLCM). All textural measures extracted from these matrices are grouped under the term "Haralick Features".

The aforementioned matrices are calculated based on the spatial domain frequency of pixels considering a pre-determined number of grey levels, which serves as the 
dimensions of the square matrix. This matrix is defined below, as:

$$
G=1\left[\begin{array}{cccc}
p(1,1) & p(1,2) & \ldots & p\left(1, N_{g}\right) \\
p(2,1) & p(2,2) & \ldots & p\left(2, N_{g}\right) \\
\vdots & \vdots & \ddots & \vdots \\
p\left(N_{g}, 1\right) & p\left(N_{g}, 2\right) & \ldots & p\left(N_{g}, N_{g}\right)
\end{array}\right]
$$

where:

- $N_{g}$ is the number of grey levels, and

- $p(i, j)$ is the probability (or count) that a pixel with value $i$ is adjacent to a pixel with value $j$.

Four variations of this matrix can be calculated by also taking the direction of adjacency into consideration. This would include horizontal $\left(0^{\circ}\right)$, vertical $\left(90^{\circ}\right)$, and the diagonal $\left(45^{\circ}\right.$ or $\left.135^{\circ}\right)$ directions. Haralick et al. [22] describe 14 textural features extracted from the basis of these matrices, which are listed along with formulae in Appendix A.

Global features describe images as a whole. Since global features embrace the advantage of a compact representation of information from the image, they are sensitive to noise and clutter. Most features that fall in this category focus on histogrambased calculations. More often than not, global features can either be built from local features or applied in a local context and overlap with local measures as well. Generally, using a combination of global and local features will aid in the improvement of object classification and the accuracy of PR [27].

Local Binary Patterns (LBP) is another textural feature extraction technique proposed by Ojala et al. [32] in 1994, which explained, in simple terms, is a thresholding approach in a local region but which represents the global image. Given a window of pixels around the focal pixel (i.e., a $3 \times 3$ window), the neighbouring pixels are assigned a ' 0 ' or ' 1 ' by achieving a comparison of the value with the centre pixel. This creates a label for every pixel, as shown in Eq. (2.2). The LBP is calculated for every pixel and compiled as a count in a histogram and used as a textural representation 
for the entire image. This can then be applied in smaller windows of the image to achieve a local feature extraction.

$$
\left[\begin{array}{lll}
51 & 54 & 78 \\
59 & 57 & 67 \\
48 & 69 & 81
\end{array}\right]=\left[\begin{array}{lll}
0 & 0 & 1 \\
1 & & 1 \\
0 & 1 & 1
\end{array}\right]=00111101=61
$$

Histogram-based calculations are very versatile in terms of the statistical measures that can be extracted as well as the pre-processing influence on an image histogram. The statistical measures include mean, variance, skewness, kurtosis, entropy, contrast, correlation, energy, homogeneity, among many others. The formation of the histograms can vary - for instance, a histogram of gradient values can be compiled where differences in pixels based on magnitudes and directions are compiled. Alternatively, histograms of an images with different applied filters can also be processed for comparison.

\section{Classification}

PR, as eloquently defined by Duda et al. [17], is "the act of taking in raw data and taking an action based on the category of the pattern". The human mind unconsciously performs this process on a continuous basis, such as recognizing our fellow peers based on their voice, or even understanding the feel of a surface without having touched it. This is possible because the object in focus (i.e., the person's voice or the visual snapshot of a surface) will belong to a certain class or category. Each class is distinctive such that it can be defined by a set of a unique combination of characteristics, or features.

When data have been gathered for the respective classes, with their respective recorded measurements or features, a training dataset has been formed whereby a pattern can be observed to differentiate between classes. This distinguishing pattern can be referred to as a classifier or more technically, a discriminant function. To solve discriminant functions for their coefficients, one can take a parametric or non-parametric approach, that is whether or not the data is assumed to have a distributional form. This type of learning and recognition is known as supervised learning, 
as class labels for existing data is a known fact.

There are different types of classifiers, such as a linear classifier (where the classification discriminant is a hyperplane) or a quadratic classifier (where the discriminant is a hyper-quadratic). Both of these essentially try to minimize the probability of classification error when computing the discriminant function, as a characteristic of the Bayesian classifier. However, they differ in the parameters assumed for each of the classes, i.e., whether the covariances are the same. The Support Vector Machine (SVM) and logistic regression algorithms are described as linear classifiers. However, SVMs are also able to achieve non-linear classification by employing a 'kernel trick', enabling the inputs to be mapped to a high-dimensional feature space. Classification models such as decision trees or artificial NNs are also commonly used, but are established schemes that can also achieve excellent classification. In the interest of brevity, we will not describe them in detail here.

$\mathrm{PR}$ and image classification, as part of the ML domain, are considered as the more intricate and complex image processing applications. When considering image classification applications deployed and used in various industries, the fundamental goal can be thought of as object recognition. After pre-processing and feature extraction, the task at hand becomes a baseline ML problem of adequate model selection for the desired prediction (categorical, continuous, clustering, etc.). We will briefly discuss the types of models that have been applied for different context goals in the image processing and classification domain.

Work on facial recognition is one of the earliest, most widely known and used image classification applications, and has been extended to analyze profiles based on behavioural observations. In 2005, Bartlett et al. [5] explored the task of recognizing facial expressions through the comparison of various ML models. The features extracted from their datasets were from a pool of Gabor filters at 8 orientations and 9 spatial frequencies, resulting in a very high dimensionality for the feature set. The tested ML models included Support Vector Machine (SVM), adaboost, and linear discriminant analysis (LDA). 
The SVM model was applied in a two-stage structure, where the first stage performed a one-versus-all classification for seven defined facial expressions and the second stage computed a probability distribution based on the previous output for the seven categories. SVMs are known to be able to handle high dimensionality in the feature space and this is further proven in the work by Chapelle et al. [9], where they explored the generalization capability for image classification using high-dimensional histogram-based features. Adaboost, while used for classification, is also used for feature selection. In this work [9], it was used to choose a subset of 900 features from approximately 166,000 features. Although the classification performance of Adaboost was comparable to that of the SVM, it was noted to be much faster due to the reduction in feature space. The LDA was tested on its own as well as with a feature space reduction using the Principal Component Analysis (PCA). However, its overall performance was not comparable to that of the SVM.

Another work towards the practical application of image classification was done by Stallkamp et al. [38] in 2012, where they aimed to benchmark ML models which classified traffic signs against human recognition. They compared results of the top three submitted models from participants against a baseline LDA classification model.

Two teams employed a Convolutional Neural Network (CNN) model, both with different structures. The first team, Sermanet [35], built a multi-scale CNN that first went through a feature extraction stage, consisting of a convolutional layer, non-linear transformation layer, and a spatial pooling layer. However, the output of both stages was fed in to the classifier - as opposed to just the second stage. The second team, IDSIA [13], used multiple CNNs as a multi-column Deep Neural Network (MCDNN). This classifier was made more robust by distorting training images during the training of the network, so that every image was distorted differently every time. The third team, CAOR [43], based their approach on the random forest ML model.

Compared to the benchmark of human recognition, only IDSIA outperformed with a Correct Classification Rate (CCR) of 99.46\%. Sermanet and CAOR fell just below the human average (98.84\%) with CCRs of $98.31 \%$ and $96.14 \%$ respectively. 


\section{$2.3 \quad$ Medical Aspects}

Diagnostic imaging in the domain of healthcare and medicine, as the process of exposing internal structures hidden by skin and bones through radiology, has advanced in the past couple of decades. Different types of medical imaging techniques include, but are not limited to, X-rays, Magnetic Resonance Images (MRIs), ultrasounds, endoscopies, etc. A wide range of pathologies can now be detected with the use of such images, depending on the bodily system under consideration (cardiovascular, respiratory, abdominal, etc.) and the imaging technique applied. Cancer, as a subcategory of these pathologies, although consisting of over 100 different types, has been a major focus point in the medicinal research community. As the cancer with the highest mortality rate, we will correlate our research in the domain of lung cancer detection and diagnosis.

The American Cancer Society estimated cancer statistics for the year of 2018, as they do every year, based on data over the past few years [37]. 234,030 new cases of lung cancer were projected, out of which $52 \%$ of cases are expected to be men and $48 \%$ are expected to be women. 154,050 deaths caused by lung cancer were projected, out of which $54 \%$ are expected to be men and $46 \%$ are expected to be women.

Lung cancer is now the second leading type of cancer for newly diagnosed patients, behind breast cancer [37]. However, as mentioned above, it has the highest mortality rate out of all cancer sites. Cancers that have been diagnosed at an early phase, such as Stage 1, can be treated with surgery and radiation therapy with an $80 \%$ success rate. Hence, the low survival rate of lung cancer patients can be associated with late diagnosis resulting in ineffective treatment due to the growth and stage of the cancer.

By nature, lung cancer is more difficult to diagnose as the most common symptoms include coughing or pain, but these occur at the later stages of the cancer. The most common tests to detect lung cancer include (but are not limited to) sputum cytology, chest x-rays, computed tomography (CT) scans, and biopsies. 


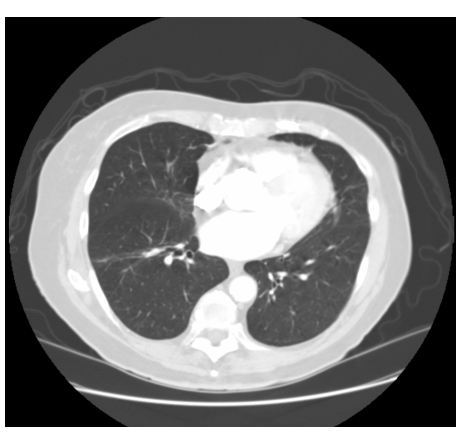

(a) Axial Plane

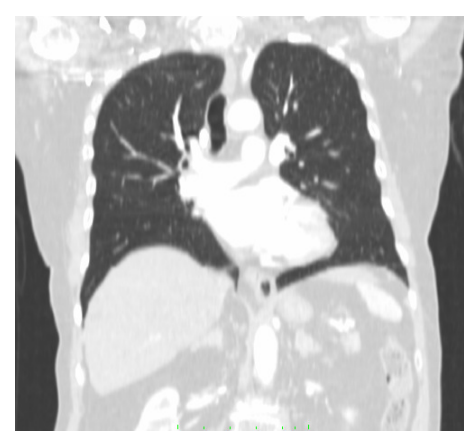

(b) Coronal Plane

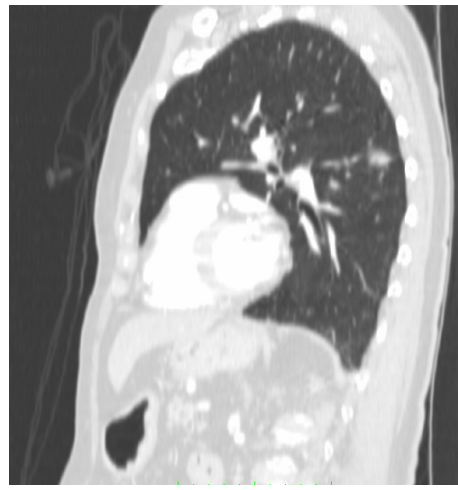

(c) Sagittal Plane

Figure 2.1: Planes captured in a computed tomography scan.

\subsubsection{Computed Tomography Scans}

The most common radiological imaging technique incorporates Computed Tomography (CT) scans where X-ray beams are used to take measurements or images (i.e., "slices") from different angles, as shown in Figure 2.1, as the patient's body moves through the scanner. Depending on the section thickness and the associated reconstruction parameters, a scan can range anywhere from 100 to over 500 sections or images [1]. The scan records different levels of density and tissues which can be reconstructed to create a three dimensional image of the human body non-invasively. A CT scan can be targeted for different parts of the body, such as the head, chest (heart and lungs), reproductive and pelvic systems, and can report various detailed pieces of information based on the contrast and exposure settings. High-resolution Computed Tomography (HRCT) is specifically used in detecting and diagnosing diffuse lung diseases [18] and cancerous nodules due to its sensitivity and specificity. It enables the detection and analysis of feature aspects such as morphological lesion characterization, nodule size measurement and growth, as well as attenuation characteristics.

\section{Hounsfield Units}

CT numbers are captured and represented as Hounsfield Units (HU) as a universally standardized dimensionless measurement. Named after Sir Godfrey Hounsfield, this 
measurement is calculated from a linear transformation of attenuation coefficients, based on the radiodensity of distilled water at standard pressure and temperature (STP):

$$
H U=1000 \times \frac{\mu-\mu_{\text {water }}}{\mu_{\text {water }}-\mu_{\text {air }}},
$$

where:

- $\mu_{\text {water }}$ : linear attenuation coefficient of water

- $\mu_{\text {air }}$ : linear attenuation coefficient of air.

Although measured in HU, CT scans and other medical imaging reports are saved in the standard Digital Imaging and Communications in Medicine (DICOM) format. There are many DICOM viewer applications specific to the domain of medicine, and useful for observing scans.

\section{Windowing in CT Scans}

As mentioned before, CT scanners record different levels of density and tissues in an organ, and they, hence, display a wide range of information dependent on the window of view. Windowing, also known as grey-level mapping, and which involves histogram modification, contrast stretching or enhancement, is the process by which the Hounsfield Units are manipulated in the grey-scale domain.

Two measures control windowing in CT scans, and they are:

- Window Width (WW) - measures the range of CT numbers (or HU) that an image contains. An image with a wide window will have a greater WW, such as 1,000. A narrow window will have a smaller WW, such as 300 .

- Window Level (WL) - measures the centre of the window width (or range).

The upper and lower grey-level bounds for the WW are calculated as follows:

$$
W W_{\text {bounds }}=W L \pm \frac{W W}{2} .
$$


The most common views observed in chest CT scans for evaluating lungs are the mediastinal and the lung window views. They, typically, have a WW and WL of $[350,50]$ and $[1500,-600]$ respectively, although these may vary between institutions and vendors. A diagram from the RadiANT DICOM Viewer Manual, displayed in Figure 2.2, shows the transition in images when manually adjusting the WW and WL. The up arrow shows the affect of increasing the window level (i.e., decreasing of brightness), and the down arrow shows the opposite. The right arrow shows the affect of increasing the window width (i.e., decreasing contrast), and the left arrow shows the opposite.

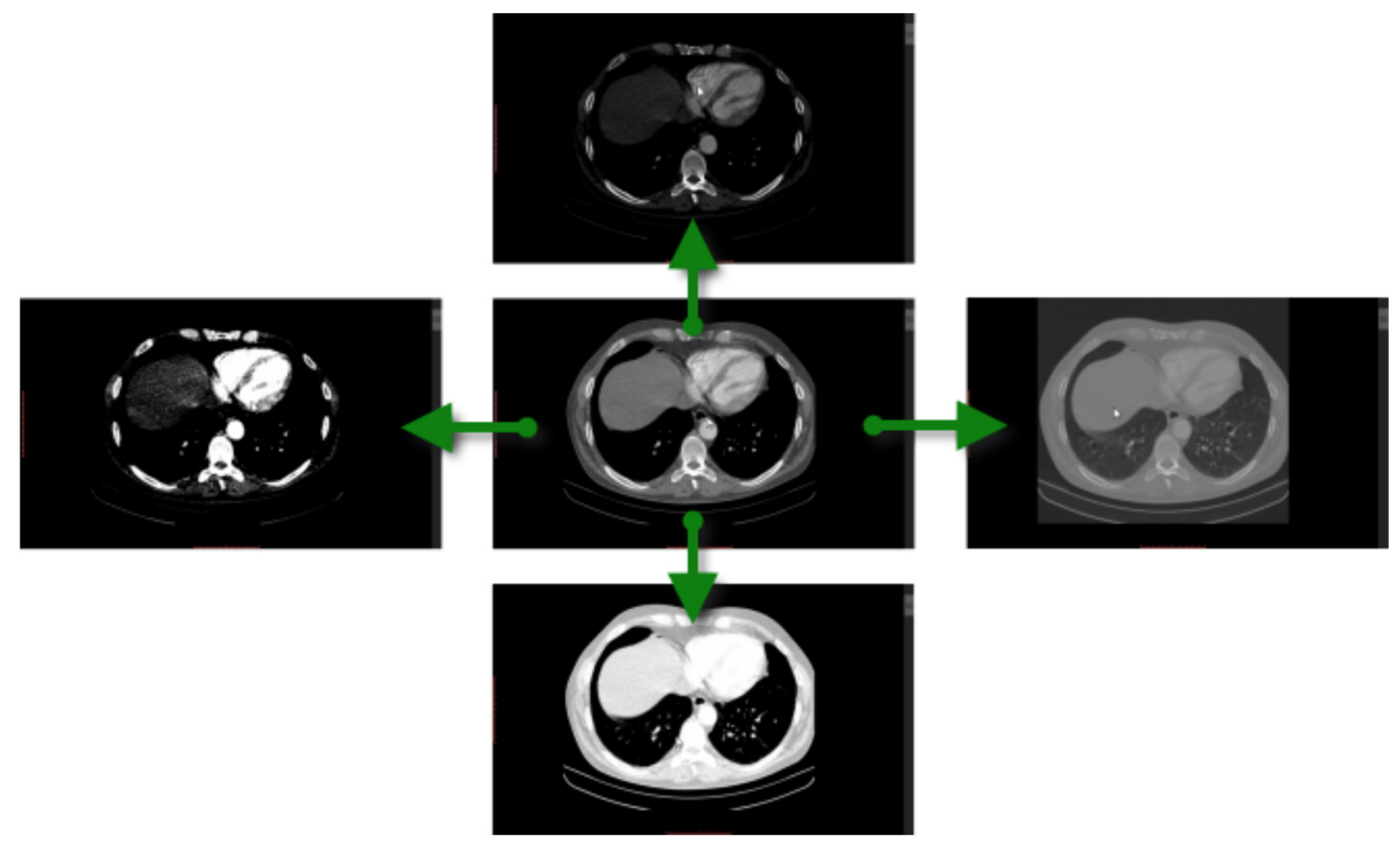

Figure 2.2: Window adjustments in Chest CT scans, sourced from RadiANT DICOM Viewer Manual. 


\subsubsection{Lung Abnormalities in Chest CT Scans}

Lungs are the main organ in the respiratory system which performs the exchange of oxygen intake to the blood stream, and carbon dioxide extraction from the blood, which is exhaled out of the system. For the purpose of this thesis, we will be exploring the analysis of a specific type of lung cancer known as adenocarcinoma, which can affect any part of the lung and is diagnosed by the detection of abnormal nodules.

\subsection{Computer Science in Medical Imaging}

Medical image processing is a research domain that has attracted much interest and research effort over the past couple of decades. The concept of a computer-aided diagnosis system was driven by the desire to use the technological benefits of digital imaging in helping radiologists with their diagnoses. Doi [16] observed that detection of potentially cancerous lung nodules is a difficult task for radiologists, with up to $30 \%$ of nodules going unnoticed due to their similarity with normal lung structures. Furthermore, general diagnostic errors with regards to CT form up to $62 \%$ of all radiology errors $[1]$.

IP techniques have been applied to a variety of radiological imaging reports, including x-rays, Magnetic Resonance Images (MRI), ultrasounds and mammograms, among many others. Although recent literature is extensively focused on integrating ML techniques to aid in the identification of specific pathologies, due to the sensitivity and impact of the healthcare domain, the majority of applications deployed and actively used by medical doctors are heavily based on IP and detection techniques (such as segmentation, area and volumetric calculations, 3D reconstructions, etc), as opposed to classification and diagnosis. This section will predominantly discuss techniques applied on Chest CT scans in the context of lung abnormality detection and diagnosis in the existing literature. However, we will also briefly review the approaches taken for other medical imaging reports as applicable. 


\subsubsection{Chest CT Scan Preprocessing}

The most popular preprocessing technique for radiographic images, is the computation and analysis of the grey-level histogram, also known as the distribution linearization technique or first order probability density function. Extracting the histogram, generally, displays values where there is a dense pixel concentration in a small portion of the histogram [21]. Applying a logarithmic conversion expands the grey range of lower-valued pixels, as most radiographic images have majority low-brightness pixels, resulting in an expansion of the contrasts. This technique is considered to be position-invariant and nonlinear, and hence pixels will remain proportional to their density as opposed to brightness.

Other preprocessing techniques include the application of varying linear filters, such as the median, Gabor, and Gaussian filters for the purpose of noise removal and image smoothing. These filters are types of low-pass filtering, which designate smooth regions but which will result in a loss of information as well. On the other hand, high-pass filters are applied where visualization of small low-contrast features [21] or image sharpness and edge detection is required for ROI extraction. Zhou et al. [45] employed a "nonlinear anisotropic diffusion filtering" technique, characterized by select partial differential equations, which helped them to achieve smoothing within regions and to retain edges between regions, thus allowing them to leverage benefits of both, low-pass and high-pass filtering.

Image subtraction is another technique employed to detect the differences in images that are fundamentally similar. However, this can only be applied to a small set of radiographic images, such as x-rays, where the difference between subsequent images does not have a substantial amount of information other than the region of interest or the abnormalities being analyzed.

\subsubsection{Lung Segmentation}

Extracting the region of interest from an image is critical for all operations that follow (i.e., image analysis, feature extraction, and/or classification). Armato III and Sensakovic [2] proposed an automated lung segmentation method for CT scans, where 

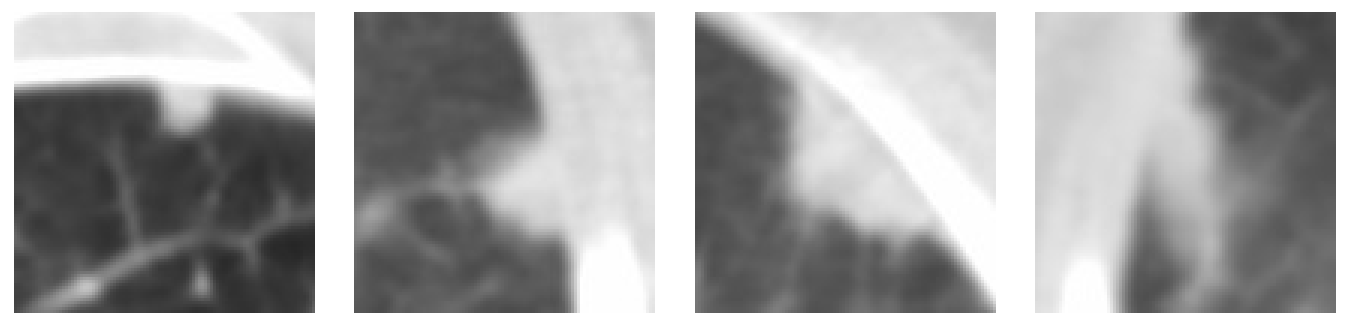

Figure 2.3: Dense structures attached to lung walls.

they focused on the inclusion of all relevant lung parenchyma for later pathology diagnosis as well as the reduction of computation time. After segmentation of the thorax - easily performed through the application of a threshold as the thorax is overlaid on a plain black background, a grey-level histogram is computed for values within this segment. Another threshold, extracted from the typically bimodal histogram, is applied to segment the lungs from other organs followed by morphological operations to "solidify" the lung. Using region-growing techniques, the trachea and bronchi are eliminated from the segmented lungs by detecting seed points from dense black regions.

However, the authors made modifications to the core segmentation algorithm after observing incorrect results [2]. As morphological operations tend to improve holes within the extracted region, dense structures (i.e., white mass) that fall along the walls (shown in Figure 2.3) of the lungs are noticeably excluded. This results in an inaccurate segmentation as these dense structures are quite often juxtapleural nodules. The modification to address this shortcoming was to apply a rolling ball algorithm, where a circular region is rolled along the lung segmentation, and matched tangentially with linear interpolation used to identify new pixels to be added to the contours. The rolling ball algorithm is a widely applied contour correction technique, adapted by many other researchers as well.

Zhou et al. [45] proposed a different technique for lung segmentation and contour correction for the inclusion of juxtapleural nodules. Following the extraction of the thorax, they identified pixels belonging to the lung through the Fuzzy C-Means (FCM) method, where the centroids are chosen based on the different tissue classes. However, for lung contour correction, they suggested an adaptive curvature threshold 
approach. This consisted of first smoothing the contour using an iterative weighted averaging, whereby a signal is smoothed by averaging the neighbourhood. This was followed by a repetitive process of measuring the height magnitudes of pixels along the smoothed contour, and connecting neighbouring pixels over a focus pixel based on the concavity curvature measure. While this approach achieved a greater level of robustness when compared to a single threshold, there was still room for improvement such as processing of sharp regions of concavity.

In relation to the aforementioned threshold-based segmentation approaches, Filippi et al. [19] explored the effects of intensity threshold selection on the segmentation and measurement of lesion volumes in multiple sclerosis (MS) in MRIs. Image intensity in MRIs, as with most radiological images, varies and depends on a number of settings in the scanner (including, but not limited to, receiver amplification factor and RF pulse tip angles). This requires the threshold selection to be a more interactive process on a patient-by-patient basis. The authors observed that a $1 \%$ change in selected threshold, resulted in a 15\% difference in computed lesion measurements and more so when the threshold varied between $2-3 \%$ of the base threshold.

Similar to Zhou et al. [45], researchers have also applied FCM as a method of segmentation on MRIs for extraction of the main tissues. Caldairou et al. [7] consider the integration of the Non-Local (NL) framework, which leveraged the advantage of repetitive structure and was initially proposed as a denoising tool. The NL framework consisted of a weighted graph computed over the similarity between voxel neighbourhoods, on which an energy function (to consider intensity inhomogeneity) and regularization (to consider image noise) could be applied for integration with the FCM.

\subsubsection{Nodule Detection and Segmentation}

Work on lung nodule detection and segmentation began decades ago, dating back to the 1990s, as the detection of such nodules is the first step to the diagnosis and treatment of lung cancer. Computer-aided techniques for nodule detection, typically, fall under two overarching categories [44]: 
1. Density-based approach - which considers the intensity of the values of the pixels in the operations applied, such as multiple thresholding, region growing, adaptive thresholding, and clustering.

2. Model-based approach - which considers the shape or physical properties of anatomical structures in techniques such as template-matching, object-based deformation, and anatomy-based generic model.

\section{Density-Based Approaches}

Zhao et al. [44] applied a density-based approach by implementing a Local Density Maximum (LDM) algorithm, which iteratively changed the threshold value in a decremental manner so as to find geometric structures that overlap with those found at the previous level. They considered a threshold step value, a threshold stop value, minimum density peak, minimum size of local maximum, as well as the ratio for volume change, as the required parameters in the LDM algorithm. Initial results yielded $94.4 \%$ sensitivity, with a high rate of 906 false-positives. This was reduced to $84.2 \%$ sensitivity after invoking modification that yielded false-positive reduction algorithm.

Similarly, Armato III et al. [4] also proposed a scheme for the detection of nodules by applying a multiple grey-level thresholding procedure. At every increment of the grey-level chosen for thresholding, the pixels that are higher than the value are labeled with an 18-connectivity parameter to create grouped structures. These structures are measured for volume, and is determined to be a nodule candidate if the volume, $V$, meets the following criteria:

$$
V<\frac{4}{3} \pi r^{3},
$$

where $r$ is the radius of the minimum enclosing sphere.

However, the goal of the multi-level thresholding was to produce a "melting" effect where structures identified at lower grey-levels disassociate into multiple smaller structures at higher grey-level thresholds. To further classify these structures as being "nodule" or "non-nodule", the authors extracted the following features to describe the structures: mean grey-level of the candidate, grey-level standard deviation, greylevel threshold for candidate identification, volume, sphericity, radius, eccentricity, 
circularity, and compactness.

Another density-based approach was discussed by Messay et al. [29], where they offered a Fully-Automated (FA), Semi-Automted (SA), and hybrid approaches for nodule segmentation. Although termed as "fully-automated", the algorithm required a single input from the user which indicated a cue-point in the nodule to be segmented, and two parameters: a threshold, $T$, and a structuring element radius, $R$. The SA approach required eight input points, and three parameters: $T, R$, and an ellipsoid scaling parameter, $E$. The hybrid approach began with the FA followed by a switch to the SA if better segmentation was required (i.e., in complex cases). The proposed algorithms employed a combination of thresholding techniques, logical (AND, OR) operations, and morphological operations to isolate the nodule from the initial bounding volume of interest around the cue point. The authors then used regression NNs as a method to evaluate the segmentations and optimize the segmentation parameters.

\section{Model-Based Approaches}

A model-based approach for the detection of initial nodule candidates was proposed by Murphy et al. [31] whereby they extracted local image features which included Shape Index (SI) and Curvedness (CV), as the interest for nodules lies in structures with a general spherical shape. These quantities are defined below:

$$
\begin{gathered}
S I=\frac{2}{\pi} \arctan \frac{k_{1}+k_{2}}{k_{1}-k_{2}}, \\
C V=\sqrt{{k_{1}{ }^{2}+k_{2}^{2}}^{2}},
\end{gathered}
$$

where $k_{1}$ and $k_{2}$ define the principal curvatures calculated for all voxels by calculating first and second order derivatives on the Gaussian filtered (i.e., blurred) image.

Seed points were then selected from the voxels by thresholding them based on the computed SI and CV values. These seed points are expanded through cluster formation and merging operations by applying further thresholding and a six-connectivity criteria. The authors then applied a two tiered k-Nearest-Neighbour (kNN) classification model on cluster features (comprising of geometric and surrounding grey values) 
as a method to reduce False Positives (FP). Cluster merging was revisited after every $\mathrm{kNN}$ classification to consider location adjustments. At four FPs per scan, the results averaged a $76.1 \%$ sensitivity for lung nodule detection.

Javaid et al. [23] employed a combination of the density and model-based approaches. The core nodule detection and segmentation algorithm was based on the density of the pixel values, following a process flow that was similar to the approaches described in the previous section. They used the K-means clustering algorithm as a baseline for nodule extraction, but also performed more operations, such as 2D morphological openings, to include nodules that were attached to blood vessel branches. The authors then proposed removal of excessive FPs from the perspective of a modelbased approach by filtering through nodule candidates based on thickness, volume, wall connectivity and sphericity.

\section{Other Approaches}

Orozco et al. [33] proposed a nodule detection technique in 2015 based on the Discrete Wavelet Transform (DWT). While most systems followed through with segmentation steps for nodule candidate detection, their suggested approach was to transform the image of the lungs to another domain. The DWT enabled a good representation of "high frequency components" (i.e., edges). Using this transform, sub-bands were extracted which contained detailed information about the vertical, horizontal and diagonal edges and structures within the image.

A more recent paper published in 2017 by Wang et al. [41] explored the application of classification models such as the CNNs for lung nodule segmentation. They considered voxel classification as a means to differentiate nodules from healthy voxels. Particularly, the authors proposed a Central Docused CNN (CF-CNN) that was adaptive for different types of nodules for heterogeneous CT data.

Their network structure included two deep (identical) branches to process 2D and 3D information. Both of these consisted of six convolutional layers, two central pooling layers, and one fully-connected layer. The convolutional layers employed kernels of size $3 \times 3$ to obtain output feature maps. The pooling layers were applied between blocks in the convolutional layers to perform feature selection on the output feature 
maps. Both branches were then combined with the connected layer where a binary softmax function was applied, from which values were converted into probability distributions for the class labels. To further clarify this, we include an illustration of this structure in Figure 2.4.

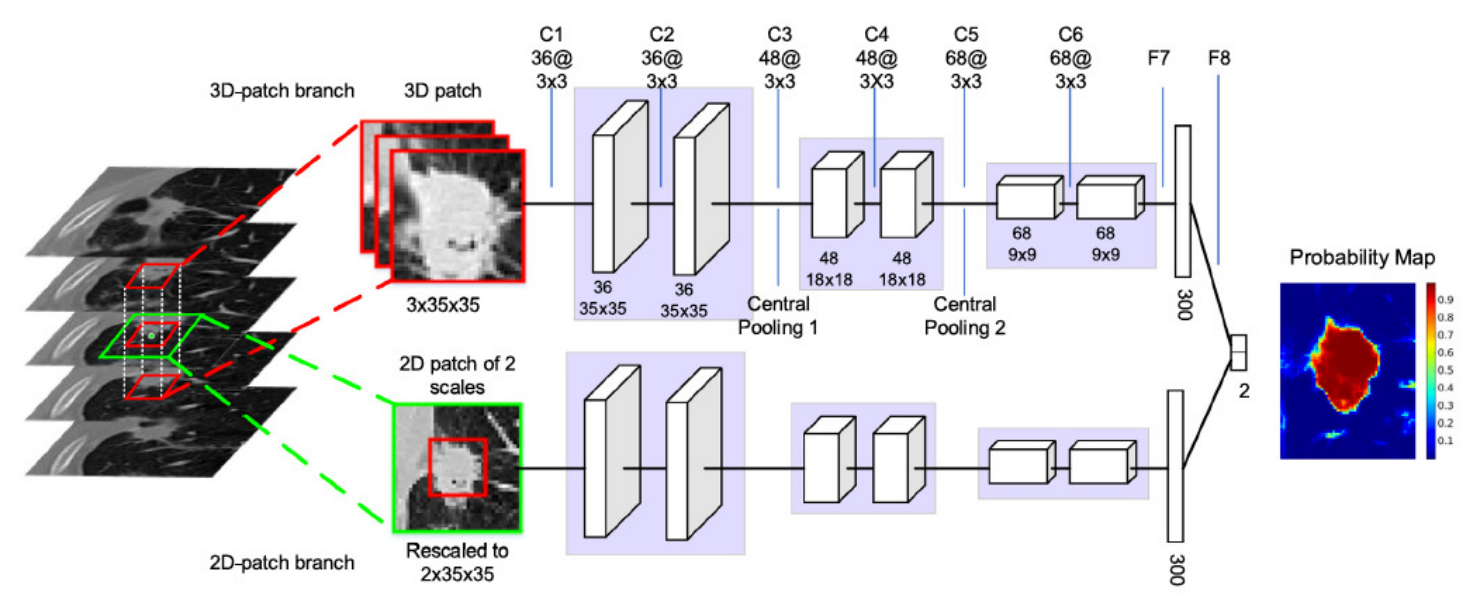

Figure 2.4: The Central focused CNN structure, including convolutional layers, central pooling and connection layer (sourced from [41]).

Using the dice similarity coefficient and the symmetric average surface distance as evaluation criteria for the segmentation, the authors observed that the central focused CNN was able to segment different types of nodules as intended and outperformed other proposed models such as the graph cut, level set, and U-Net segmentation, validated with visual evidence.

\section{The Lung Nodule Analysis 2016 (LUNA) Challenge}

The LUNA16 Challenge was a grand challenge introduced in 2016, that has been closed for submissions since January 2018. In the challenge, participants were invited to conduct research on an open $\mathrm{CT}$ dataset, provided by the Lung Image Database Consortium image collection (LIDC-IDRI), that has been annotated for lung nodules in 3 categories based on the size of the candidate and the classification of nodule or non-nodule. Two tracks were offered for participation: complete Nodule Detection (NDET), and False Positive Reduction (FPRED). The organizers, Setio and et al. 
[36], compiled submissions and published a paper in 2017 reviewing techniques that were applied by participants.

Combining multiple algorithms from the submission pool for nodule candidate detection achieved a sensitivity of $98.3 \%$ where the best single submission had achieved a sensitivity of $92.9 \%$ through the application of enhancement filters and the divergence of normalized gradient for centre detection. The best submissions for NDET and FPRED, judged predominantly by FROC curves, were both techniques that employed a CNN for nodule classification. However, it was noted that any combination of the proposed systems achieved a higher sensitivity than the best system.

\subsubsection{Nodule and Texture Feature Extraction}

Feature extraction schemes in biomedical applications have been found to be specific to the context of the goal at hand. However, it may be beneficial to derive a wide variety of features and to then reduce the set to those which prove to be most relevant [21]. This can be done through the application of feature elimination or feature selection techniques.

The goal of forming a descriptor vector in the context of nodules and texture, is a task of local feature extraction. Chabat et al. [8] and Kim et al. [25] aimed to classify obstructive lung diseases based on texture patches as regions of interest (ROI). For each ROI, a statistical descriptor was calculated to describe the CT attenuation

characteristics. The following list of textural features were widely used across the literature in this domain:

- Typical histogram and gradient features such as mean, standard deviation, skewness, and kurtosis;

- Energy: ene $(C)=\sum_{i, j} C_{i, j}{ }^{2}$;

- Entropy: $\operatorname{ent}(C)=-\sum_{i, j} C_{i, j} \log C_{i, j}$;

- Maximum: $\max (C)=\max C_{i, j}$; 
- Contrast: $\operatorname{con}(C)=\sum_{i, j}|i-j|^{\alpha} C^{\beta}{ }_{i, j}$ where $\alpha=1$ and $\beta=1$;

- Homogeneity: $\operatorname{hom}(C)=\sum_{i, j} \frac{C_{i, j}}{1+|i-j|}$;

- Short primitive emphasis: spe $=\frac{1}{B_{t o t}} \sum_{\alpha=1}^{p} \sum_{r=1}^{r_{\max }} \frac{B(a, r)}{r^{2}}$;

- Long primitive emphasis: lpe $=\frac{1}{B_{t o t}} \sum_{\alpha=1}^{p} \sum_{r=1}^{r_{\max }} B(a, r) r^{2}$;

- Grey-level uniformity: $g l u=\frac{1}{B_{t o t}} \sum_{\alpha=1}^{p}\left[\sum_{r=1}^{r_{\max }} B(a, r)\right]^{2}$;

- Primitive length uniformity: $p l u=\frac{1}{B_{t o t}} \sum_{r=1}^{r_{\max }}\left[\sum_{\alpha=1}^{p} B(a, r)\right]^{2}$.

In the above, the variables are as follows:

- $C$ : A texture patch of arbitrary dimensions, $d \times d$;

- $r_{\max }$ : Maximum primitive length;

- $B_{t o t}$ : The total number of acquisitions;

- $B(a, r)$ : The number of primitives of all directions having length $r$, and graylevel $a$.

However, Kim et al. [25] also included other features such as the co-occurrence matrix and the top-hat transform. Additionally, they incorporated measurements to depict shapes in the ROIs, such as circularity and aspect ratios, which were not well represented by statistical measures extracted for texture. Similarly, Demir and Camurcu [15] expanded the aforementioned list with morphological features to describe the shape of nodules in the ROIs.

Ramalho et al. [34] proposed the processing of a spatial interdependence matrix (SIM) as a representation of structures in the lungs using three values to classify chronic obstructive pulmonary diseases (COPD). The SIM was defined by considering 
an image, $I$, and its degraded version, $J$, with $N$ grey levels, whereby intensity transitions, $M$, between the images were captured as:

$$
M_{i j}=\#\{(i, j): i=I(p), j=J(p)\}
$$

where $\#\{\cdot\}$ stands for the cardinality of the set of intensities. From this matrix, three values were computed, namely correlation (Cor), inverse difference moment (Idm), and chi-square (Chi), which were defined as follows:

$$
\begin{gathered}
C o r=\sum_{i, j=0}^{N-1} \frac{\left(i-\mu_{i}\right)\left(j-\mu_{j}\right) M_{i j}}{\sqrt{\sigma_{i}^{2} \sigma_{j}^{2}}} \in[-1,1], \\
I d m=\sum_{i, j=0}^{N-1} \frac{M_{i j}}{1+|i-j|} \in[0,1], \\
\text { Chi }=\sum_{i=0}^{N-1} \frac{\left(O_{i}-E_{i}\right)^{2}}{E_{i}} \in[0,1],
\end{gathered}
$$

where:

- $\mu_{i}$ and $\mu_{j}$ are the average values;

- $\sigma_{i}$ and $\sigma_{j}$ are the standard deviations of the normalized $M$;

- $O_{i}$ is the observed weight in the diagonal $(i=j)$ of $M$;

- $E_{i}$ is the expected weight in the diagonal of $M$ for $J=I$.

These features were compared against those extracted from the Grey-Level Cooccurrence Matrices (GLCM) as well as visual information fidelity index on the same images, and it was observed that the SIM features provided the best discrimination.

In the context of MRIs, Wang et al. [42] explored the extraction of information to form continuous clinical variables using high-dimensional PR. The authors addressed the challenge of dimensionality reduction in image-based ML through the analysis of the discrimination ability of adaptive regional feature clustering. Corresponding to brain MRIs, they calculated different tissue density maps for grey matter, white 
matter and spinal fluid as a voxel measurement to compare with regressed clinical scores as opposed to class labels. These features were followed by a Relevance Vector Machine, which proved to overcome the shortcomings of using the SVM as a regression model.

\subsection{Chapter Conclusions}

In this chapter, we conducted a survey of the field of medical image processing and the existing ML applications in the context of various medical applications. By all metrics, the survey was necessarily brief, although for our purposes, it was fairly comprehensive. We considered the topic of ML in the context of images, i.e., the feature extraction process starting with the pixels in an image. This included the published techniques to obtain local and global features, and the suitable methods of classification for image-based PR. Additionally, we explained the background of medical imaging, and more specifically CT scans and its technical aspects such as the measure of attenuation (HU) and the concept of "windowing" to control contrast adjustments.

The second half of the survey discussed the state of the existing research for the application of ML in medical imaging and diagnosis. This consisted of IP techniques used for medical images, as well as the types of features extracted and the models designed for various medical contexts. We observed that most of the research conducted has concentrated on classification-based goals - such as the presence of a cancerous nodule, or the automated segmentation of lungs and nodules. However, very few papers have reported work within the context that was more clinically applicable for the prediction of survival durations. In our work, we will evaluate the severity of a pre-diagnosed lung cancer through the prediction of survival times which can, further, affect the prognosis and treatment of the illness. 


\section{3 \\ Lung Abnormality Detection}

\subsection{Introduction}

This chapter will focus on a prior algorithm that will be used as the basis for this thesis, which is essentially a foundational prototype for a Computer-Aided Diagnosis (CAD) System. The latter has incorporated within it a complete implementation of the detection module of a CAD system. We will first review the methodology and process flow of this algorithm, the image processing techniques employed, and the additional work required to ensure the correctness of the work done in this thesis.

\subsection{Foundational Algorithm}

The algorithm alluded to above is a work that was previously done by the author as a research-based capstone project for the completion of an earlier degree program. 
The algorithm was initially prototyped using MATLAB's Image Processing Toolbox. Since the inception of this research, the algorithm has since been ported to Python for more flexibility. A literature review of the published work in the domain of medical image processing provided a variety of proposed methodologies that could have been followed for the classification of a lung in a Chest CT scan. However, from our investigations, we determined that the existing research has been focused on the detection of specific pathologies and the processing of localized regions in the scans.

The prior algorithm, however, performed a more generalized output in the form of a binary classification where it can also detect a range of abnormalities. Although localized processing is vital for the detection of abnormal tissue and texture, it would be infeasible to utilize the results to provide a holistic diagnosis of the entire lung. On the other hand, merely extracting those features that would allow for the diagnosis of the lung on a global scale also seems to be a more simplistic and narrow approach due to the vulnerability of missing vital details. Consequently, the algorithm focused on an intermediary scale, i.e., the slice of a scan. Rather than classifying an entire lung as being 'Healthy' or 'Unhealthy', or classifying a small texture patch as 'Normal' or 'Abnormal', the system classified slices of a Chest CT scan as 'Healthy/Normal' or 'Unhealthy/Abnormal'. This approach utilized the benefits of both, global and local processing, and was implemented in a 2-tier model. Among the extracted features, most of them were based on the region of the lung in the given slice, whereas others, such as the texture, were based on localized areas. The texture as a feature, however, has also been determined through a classification procedure. Extracted lung images were split into patches of texture and passed through a model trained on a library of patches marked as 'Normal' and 'Abnormal'. Traits and statistics of the abnormal patches, if any, were returned as descriptors for the texture feature. All the features were then used to describe a single slice, which were passed through a classification model trained on a library of slices from the scans.

Figure 3.1 shows a process diagram describing the overall flow of the algorithm. It has been divided into 5 major steps: receiving an input, preprocessing, feature extraction, classification, and emitting an output. The main components and the respective details of the system flow are as follows: 


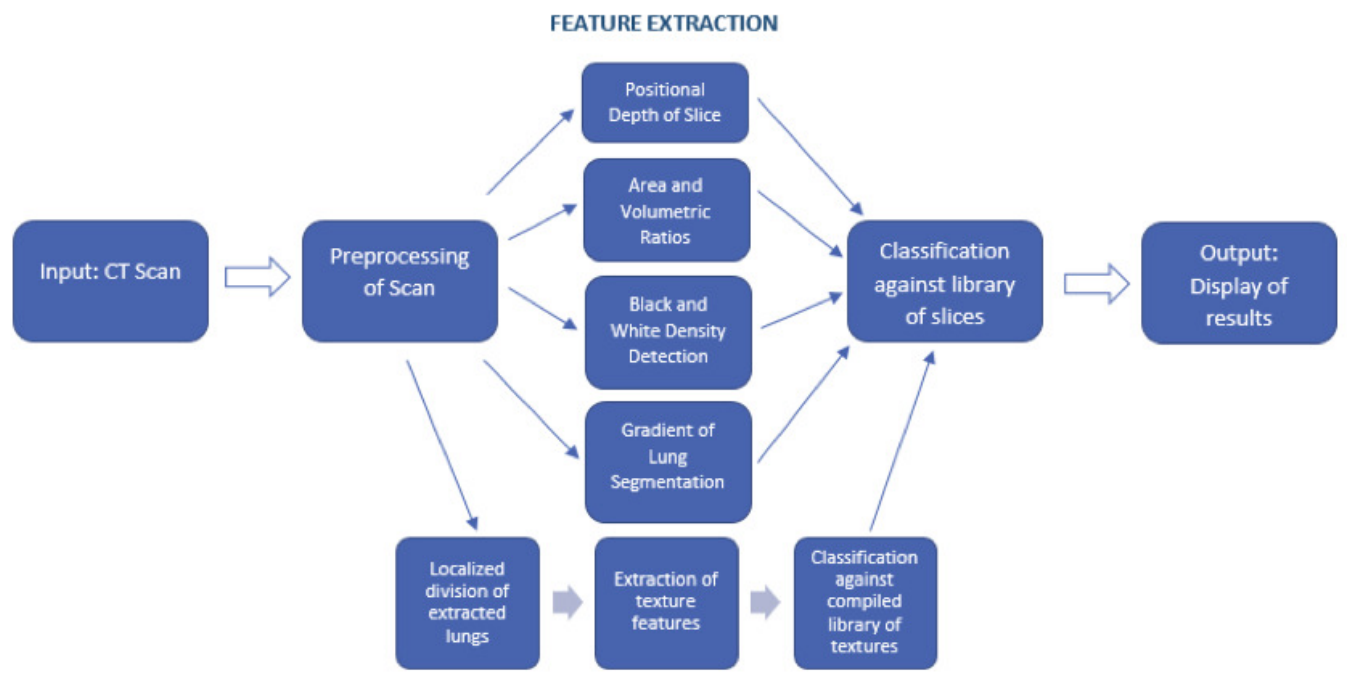

Figure 3.1: Process flow of the prior abnormality detection algorithm.

- Preprocessing: This implemented several operations such as:

- Intensity adjustments for switching to and from lung window and mediastinal views;

- Lung segmentation to extract the region of interest.

- Feature extraction: This involved extracting information to be used as descriptors to represent the lung being evaluated. The features varied in nature, as some of them were computed globally, some were computed regionally (i.e., within a single slice), and others were computed locally, as those listed below:

- Global - the position of the slice (i.e., the depth of the slice in relation to the lung);

- Regional - ratio calculation: area/volume of abnormalities to normal lung;

- Regional - detection of dense consolidation;

- Regional - detection of abnormal air; 
- Local - texture analysis for pattern and intensity distribution and quantification.

- Feature selection: In this case, it invoked a feature selection process to remove weak descriptors from the extracted features, especially on the local level.

- Classification: In this case, multiple classification models were implemented and tested using the selected features.

We will discuss the methods that were implemented to achieve the desired results of each stage of processing. For the sake of illustration and understanding, we will also display the intermediate images from the dataset after invoking the corresponding processing steps.

\subsubsection{Preprocessing}

As mentioned in Section 2.3.1, working with the images of chest CT scans means processing data in the DICOM format. This storage format essentially groups information in the datasets, enabling the attachment of patient and pixel data, as well as technical data, such as the corresponding encoding schemes and window measurements, through attribute tags.

The DICOM images are expressed as 16-bit integer values, where the stored attribute tags specify the default, and include:

- Slice thickness;

- Number of rows and columns (i.e., image dimensions);

- Window centre and window width, as defined in Section 2.3.1;

- Rescale intercept, $m$, and rescale slope ${ }^{1}, b$, which are used in the linear conversion of the stored value, $S V$, to the appropriate Hounsfield Unit (HU), $U$ :

$$
U=m S V+b .
$$

\footnotetext{
${ }^{1}$ It is important to note that more often than not, the rescale slope was valued at 1 . Indeed, we have not encountered a dataset which has a different rescale slope value.
} 


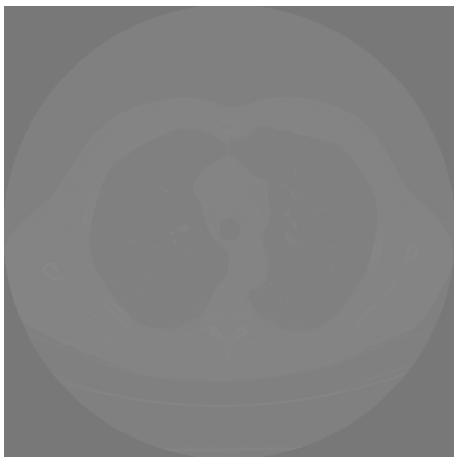

(a) Default View

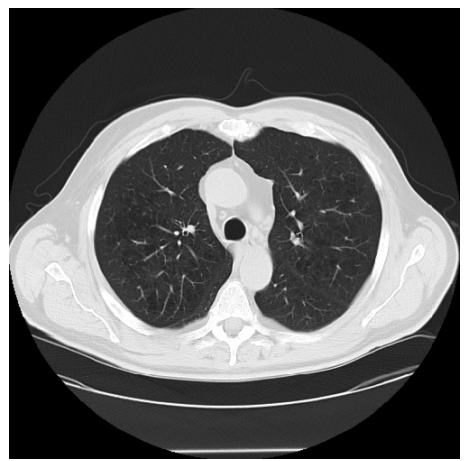

(b) Lung Window View

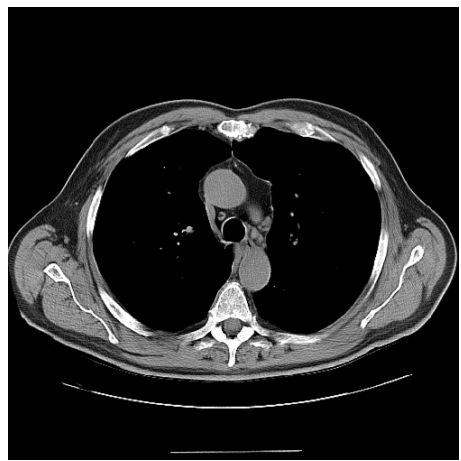

(c) Mediastinal View

Figure 3.2: Different Chest CT views based on window parameter adjustments.

As part of the preprocessing stage, the images, originally displayed as shown in Figure 3.2a, are first converted to be representative of the HUs using Eq. (3.1) for easier processing and visibility. Images are then scaled to two different window specifications for the Lung Window view, also known as the Pulmonary view, and the Mediastinal view, respectively. The Lung Window view, shown in Figure 3.2b, displays the texture in the lung and is attained by adjusting the window centre and window width, $[C, W]$, parameters to $[-500,1400]$. The Mediastinal view, shown in Figure $3.2 \mathrm{c}$, is attained by adjusting the parameters to $[40,380]$.

\subsubsection{Lung Segmentation}

The next stage involved the extraction of the ROI which is done by segmenting the lungs. A general thresholding approach was used for the lung segmentation. This was achieved by using the mediastinal view, since the lungs are dense black regions, and applying a global threshold to separate the body and miscellaneous components included in the CT images, such as the CT bed. By finding statistics of connected components in the binary image, components that are smaller than the size of the largest component (i.e., the body of the patient) are then removed.

Left with just the body of the patient, the morphological operation known as "closing" is applied to close small holes within the body component that were excluded from the global threshold, yielding Figure 3.3a. Inverting the binary image and 


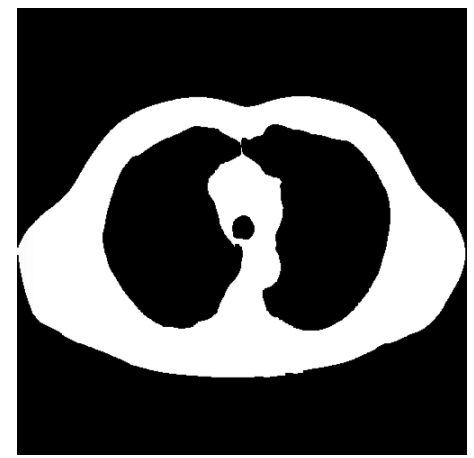

(a)

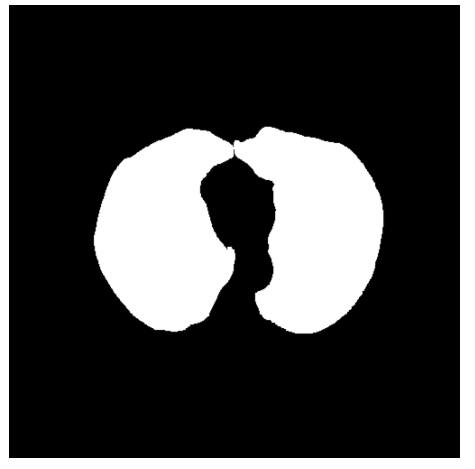

(b)

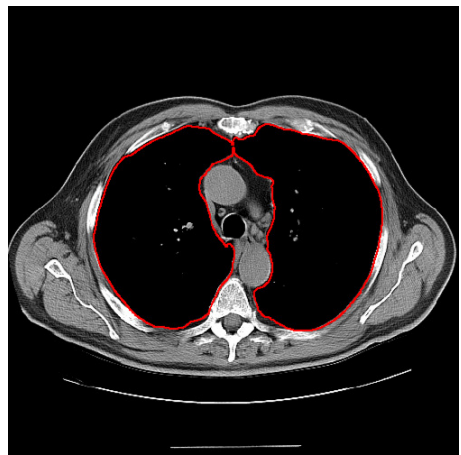

(c)

Figure 3.3: ROI extraction.

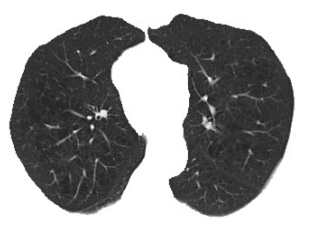

(a)

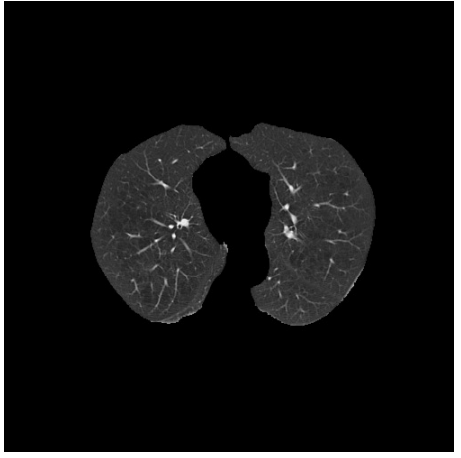

(b)

Figure 3.4: Lung extraction.

removing the largest component (i.e., the background), and components smaller than a chosen threshold to remove the windpipes, returns the lungs as shown in Figure $3.3 \mathrm{~b}$, which will, hereafter be referred to as the "mask" of the lungs. The boundary is extracted using the Canny edge detection technique, and can be overlayed on the original image for visual understanding (Figure 3.3c). The mask is then multiplied ${ }^{2}$ against the original image of the lungs to extract the real lungs against a plain white or black background as desired, as shown in Figure 3.4.

\footnotetext{
${ }^{2}$ The algorithm invokes an element-wise matrix multiplication. Since the mask is binary, and only consists of 1's and 0's, the actual operation that takes place here is, precisely, that of a logical AND operation.
} 
For the purpose of the preliminary capstone project, this method of lung segmentation was adequate for the overall detection of abnormalities. However, for the work done in this thesis, we require additional significant modifications due to the nature of the juxtapleural nodules and their physical attachments to the lung wall. This is further discussed in Section 3.3.

\subsubsection{Feature Extraction}

With the image of the lungs being extracted, which resulted in a reduction of the processing area, the focus of the algorithm was to obtain meaningful information which was to be used to constitute the features. As the initial goal was a simple binary classification, we concentrated on the general form of structures which could indicate a variety of pathologies. These included detecting a stark difference in the area and volumetric ratios when compared to those of a normal lung, detecting irregular dark (almost black) regions in the texture thus indicating the presence of abnormal air, and detecting irregular masses depicted with regions of a lighter grey shade in the texture, which further signified the presence of a form of dense consolidation.

\section{Area and Volumetric Ratios}

Quantitative measures included as part of the feature set were the measurements of area and volumetric ratios of air in the lung to tissue in the lung (i.e., blood vessels and abnormal masses). The calculated ratios were indicators for cases where there was significant pleural interruption or abnormal masses in the lung. The area ratios were specific to each slice (or image) in a scan, whereas the volumetric ratio was a global measurement and correlated to the entire lung.

The area measurements were calculated by thresholding the extracted mediastinal lungs to binarize the image, such that any tissue found in the lung was rendered to be white. It is important to note that the expected ratio was not 0 due to the fact that blood vessels were included in the threshold of the tissue matter, and thus, until a certain limit was obtained, they were considered normal. The statistics of all whiteconnected components were then obtained, and the area of all connected components 
was summed and stored as the area of the tissue matter.

A similar process was applied to the image containing the mask of the lungs (Figure 3.3b), giving us the total area of the lungs. Dividing these values yielded the desired ratio. The volumetric ratio was calculated by dividing the computed sum of the area measures over all the images in a single scan.

For instance, if we consider the lung shown in the previous sections, which is depictive of a normal lung image, the area measurements and ratios are as follows:

- Tissue Mass: 214 pixels;

- Lung Mass, inclusive of tissue mass: 51,306 pixels;

- Ratio, calculated by dividing tissue mass by lung mass: 0.004755 .

\section{Abnormal Air and Dense Consolidation Detection}

The next features were based on the presence of densely dark (abnormal air) or densely light (dense consolidation) regions in a lung image. Abnormal air is, essentially, any pocket of air in the lung that forms a type of bubble including abnormal vessels or abnormal tissues around the bubble. Dense consolidation is any type of aggregated mass (vessels or tissues) that is seen in the lungs. It is not uncommon for the scan to display dense consolidation at the edges of an abnormal air pocket.

Using the image of the textured lungs on a white background (Figure 3.4a), a very low threshold value was applied for the extraction of any dense dark regions in the lung. Similarly, using the image of the textured lungs on a black background (Figure 3.4b), a relatively high threshold value was applied for extraction of any dense light regions in the lung. The boundaries were then extracted so that they could be superimposed on the original image, as well as the area of the detected region, if present, as shown in Figure 3.5.

\subsubsection{Texture Analysis}

As explained earlier, the texture was analyzed on a local scale where patches fully containing lung matter were extracted from the segmented lungs. An appropriate 

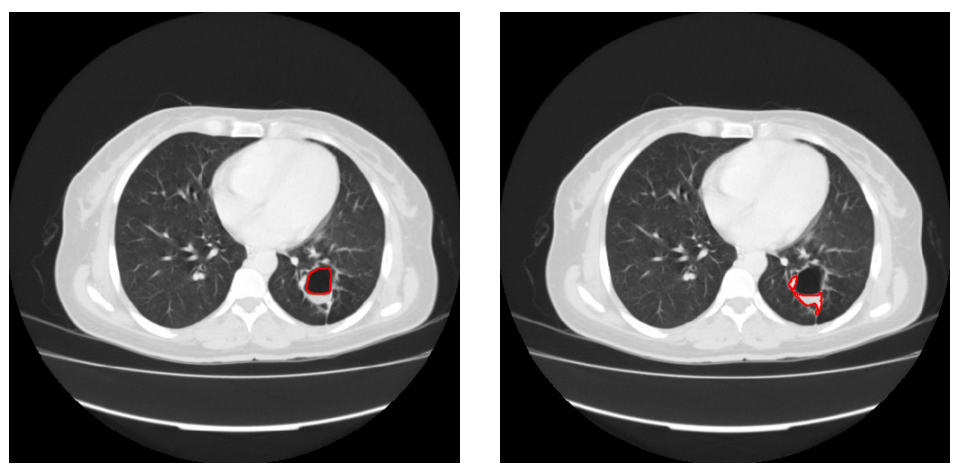

Figure 3.5: Detection of abnormal air and dense consolidation regions.

size for patches was determined through a trial-and-error process. The first patch size tested was 25 x 25 pixels, which conclusively, was not large enough to encompass abnormal structures in the lung, thus creating a large overlap between the set of normal and the set of abnormal patches. The second patch size tested was $49 \times 49$ pixels. It is important to note that the size of the patch taken had to be an odd number so that a centre pixel would be present for the purpose of multiple image processing operations. However, the second patch size proved to be larger than the requirements that were initially proposed, especially due to the detrimental effect on performance and running time of the algorithm. This led to an intermediary size of 37 x 37 pixels, which was large enough to contain abnormal structures and in turn, decreased the overlap between the normal and abnormal sets.

Patches from an image can be extracted in one of two ways, distinctly or by sliding. In distinct patches, pixels that have been grouped into one patch cannot be included in another patch. On the other hand, in the sliding method of extraction, patches are stored as if a sliding block passed over the image with a patch fitting to any group of pixels that fit in the block, regardless of its membership in another patch. The resulting number of patches from "sliding" versus "distinct" extraction is significantly more, expressed by the following equation:

$$
p=(q-m+1)(r-n+1),
$$

where:

- $p$ : number of patches; 
- $[m, n]$ : dimensions of the image;

- $[q, r]$ : desired dimensions of the patches.

Using "sliding" patches was the chosen approach for localized texture processing, as this ensured the most coverage of the lung matter given its irregular shape. For this reason, the resulting number of patches from a single image slice in the CT scan was not quite in accordance with Eq. (3.2), as any patch with a plain white background was discarded for analysis. This aspect, however, required modifications due to the nature of adenocarcinoma tumours as juxtapleural nodules attached to the lung walls (to be discussed further in Section 3.3). Furthermore, in the case that a patch was classified as being "Abnormal", the chances of subsequent patches (i.e., those that had a significant overlap of pixels) being classified as "Abnormal" would also be quite high, thus providing a more contiguous region of abnormal texture.

Once the patches were extracted, statistical texture features were computed as a quantitative representation of the patch. While the algorithm was in MATLAB, the texture features included the properties of the Grey-Level Co-occurrence Matrix (GLCM), previously defined and explained in Section 2.2.1, which included contrast, correlation, energy, and homogeneity - were all computed using available API calls in the MATLAB Image Processing Toolbox (IPT). Additionally, the Grey-Level Run Length Matrix (GLRLM) and its relevant properties were also computed, and the indices obtained including the following:

- Short Run Emphasis (SRE);

- Long Run Emphasis (LRE);

- Grey Level Non-Uniformity (GLN);

- Run Percentage (RP);

- Run Length Non-Uniformity (RLN);

- Low Grey Level Run Emphasis (LGRE);

- High Grey Level Run Emphasis (HGRE). 

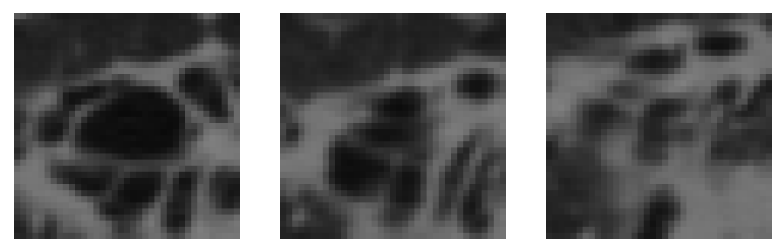

Figure 3.6: "Abnormal" texture patches.

The aforementioned statistical measures constituted a 13-dimensional feature vector. However, the feature selection process excluded all seven GLRLM properties, as it had a negligible effect in the accuracy of the tested model(s), reducing the dimensionality of the feature space. A notable drawback of the remaining measures was the running time, as the computation of the GLCM was quite costly.

Once the algorithm was ported to Python, the OpenCV-Python library was used to implement the IP operations. However, for texture feature computations, we invoked the Mahotas library [14]. We calculated a 12-dimensional feature vector using the Haralick features, defined in Appendix A. The models tested on both the MATLAB and the Python algorithm, are discussed in the next section.

\subsubsection{Results}

The prior algorithm attempted a simplistic 2-tier approach by performing a classification on the extracted texture features whose output was used as a part of the feature vector in the classification on the image of the lung. We will briefly discuss the results of the ML models applied, and the drawbacks of the prior algorithm.

\section{Tier 1 - Texture Prediction}

To build models for a binary classification of the texture present in the lung, a texture patch library was manually compiled using feedback and annotations from the partnering radiologists. Scripting with MATLAB, the author iterated through the images of the scans in the dataset and picked patches to accurately portray what should be considered "Abnormal" (as shown in Figure 3.6) versus what should be considered "Normal" (as shown in Figure 3.7). 

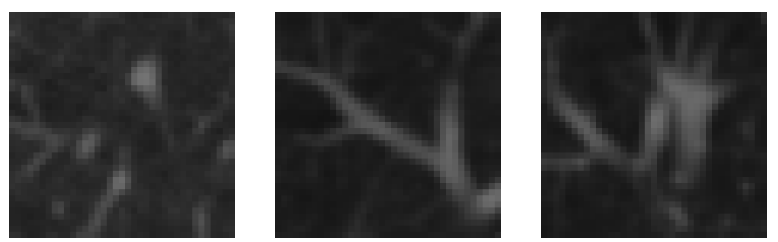

Figure 3.7: "Normal" texture patches.

For the MATLAB version of the algorithm, developed on MATLAB 2013a, we tested the data on the classification ML schemes which included a Decision Tree (DT) model, an SVM model, and a NN model ${ }^{3}$. These were tested incrementally while the texture library was being compiled in order to observe the effect of the library size. Unfortunately, the only documented evaluation metric was the accuracy of the models, and the results are listed in Table 3.1. As one observes, the NN model yielded the highest accuracy for the library of 1,000-patch sizes. However, those patches were extracted from only a fraction of the scans present in the dataset, and hence, were assumed to not be a complete representation of the different "Abnormal" textures.

Table 3.1: MATLAB Classification Model Accuracies

\begin{tabular}{c|ccc}
\hline \hline Library Size & DTs & NNs & SVM \\
\hline 1000 & $87 \%$ & $96 \%$ & $91 \%$ \\
2120 & $89 \%$ & $84 \%$ & $93 \%$ \\
3240 & $93 \%$ & $50 \%$ & $95 \%$ \\
\hline
\end{tabular}

For the Python version of the algorithm, using the scikit-learn ML library, we tested the following classification models on the 3,240-patch library: DT, Gaussian Naive Bayes, $k$-nearest neighbours $(\mathrm{kNN})$, Logistic Regression, and the SVM. The models were tested on the feature set constructed from the MATLAB IPT, as well as the Mahotas image processing library for Python. The evaluation metrics included accuracy, precision, and recall, which are listed in Table 3.2. The highest metrics were for the DT model with the Mahotas-Haralick features.

\footnotetext{
${ }^{3}$ The NN model was a feed-forward algorithm with a given number of hidden layers, where the weights were trained using the so-called "Bayesian Regularization" training algorithm.
} 
Table 3.2: MATLAB Classification Model Accuracies

\begin{tabular}{l|l|ccc}
\hline \hline & & \multicolumn{3}{|c}{ Evaluation Metric } \\
Feature Set & Classification Model & Accuracy & Precision & Recall \\
\hline \multirow{3}{*}{ MATLAB IPT } & DT & $89.5 \%$ & $93.0 \%$ & $93.7 \%$ \\
& Gaussian Naive Bayes & $84.2 \%$ & $93.0 \%$ & $85.2 \%$ \\
& kNN & $91.7 \%$ & $93.9 \%$ & $95.1 \%$ \\
& Logistic Regression & $90.8 \%$ & $91.4 \%$ & $96.8 \%$ \\
& SVM & $90.1 \%$ & $91.9 \%$ & $95.1 \%$ \\
\hline \multirow{5}{*}{ Mahotas Haralick } & DT & $96.3 \%$ & $97.7 \%$ & $97.4 \%$ \\
& Gaussian Naive Bayes & $88.9 \%$ & $93.4 \%$ & $92.3 \%$ \\
& kNN & $88.0 \%$ & $89.3 \%$ & $96.1 \%$ \\
& Logistic Regression & $91.5 \%$ & $92.1 \%$ & $97.6 \%$ \\
& SVM & $77.6 \%$ & $77.6 \%$ & $100 \%$ \\
\hline
\end{tabular}
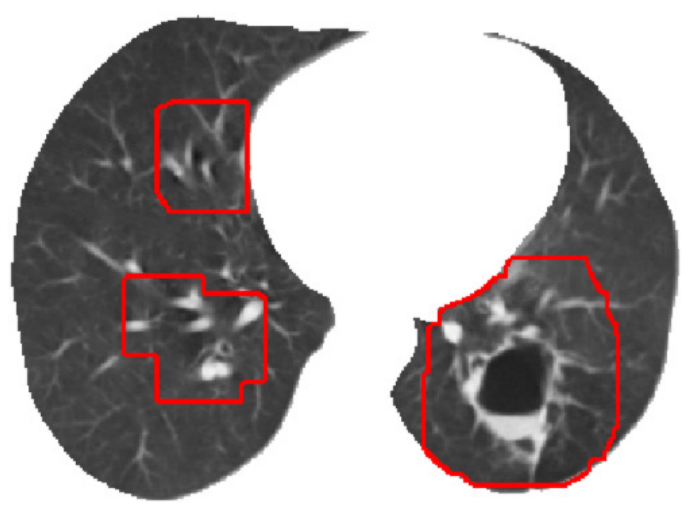

Figure 3.8: Highlighted abnormally classified texture patches.

The patches that were then categorized as "Abnormal" were marked on the lung, and as mentioned earlier, the trend for detection of contiguous regions of patches to be marked as "Abnormal" was observed. Consequently, the boundary of the entire region was superimposed onto the image, as opposed to highlighting every patch, as shown in Figure 3.8.

\section{Tier 2 - Slice Image Prediction}

The goal for the prior algorithm was to pass the global feature set, including the positional depth of the slice, area and volumetric ratios, abnormal air and dense 
consolidation detection, as well as the percentage and area of abnormally classified textures, through a subsequent classification model. However, due to inconclusive results and time constraints, the brief testing of the models did not yield plausible results. Consequently, in the interest of terminating the prior project, an if-else structure was implemented to consider the presence of abnormal air, dense consolidation, or abnormal texture. This was utilized as an indicator for deciding whether the slice had to be considered "Abnormal".

\subsection{Additional Work}

While the prior algorithm had a general focus on whether abnormalities were present in the lung, the work of this thesis is specific to nodules already known to be present in the lung and that have already been classified as the most common type of lung cancer, i.e., pulmonary adenocarcinoma. This further restricts our ROI as well as the processing area when it concerns the task of feature extraction.

\subsubsection{Lung Tumour Segmentation}

As mentioned in Section 3.2.2, the segmentation performed in the existing algorithm excluded juxtapleural nodules, i.e., those that are attached to the lung wall. Many of the nodules in the data set considered for this thesis involved cases of this type. One modification that could be made to the prior algorithm's segmentation methodology would be to implement the so-called "rolling ball" algorithm for lung boundary correction so as to include juxtapleural nodules [3]. However, this approach would not have been able to guarantee accurate and dependable results for all scans.

As the new focus does not require the entire lung region, we opted to take an alternate approach by making masks of the tumours using the ImageJ software ${ }^{4}$. This was done by manually tracing a contour around the nodule on the images where it was present, filling the shape as "white", and clearing the background to "black". The images that did not contain the nodule were cleared to a "black" background.

\footnotetext{
${ }^{4}$ The ImageJ Software is a Java-based image processing program developed at the National Institutes of Health and the Laboratory for Optical and Computational Instrumentation.
} 


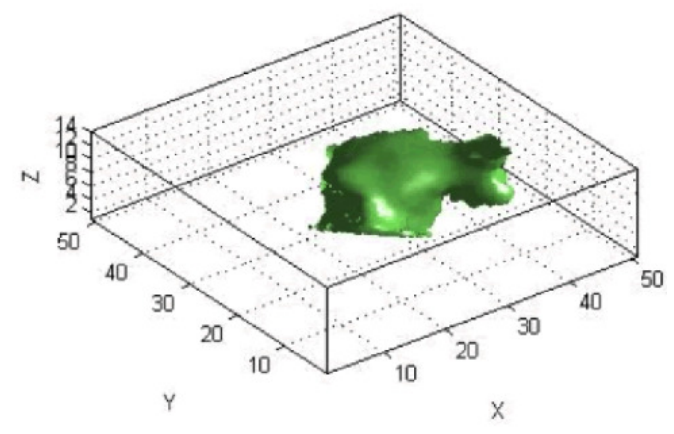

Figure 3.9: 3D visualization of tumour nodule, sourced from [39].

\subsubsection{D Nodule Construction}

Segmenting the scans manually for nodules created a mask for each scan in the dataset. This enabled a simpler implementation of the algorithm to extract the ROI. For each scan, the respective segmentation mask was loaded, and all the images were re-iterated. If a connected component was found (signifying the presence of a nodule) in a mask image, the original image was operated on by a pixel-wise multiplication, which, as before, turns out to be the same as invoking a bit-wise logical AND operator, so as to precisely extract the nodule. In this manner, we were able to obtain the 3D matrix of the nodule in a scan by stacking the individual images, similar to the process implemented by Senthil Kumar et al. [39] as shown in Figure 3.9, which can be further used for visualization and feature extraction.

\subsection{Chapter Conclusions}

In this chapter, we reviewed the previously prototyped CAD algorithm on which this thesis is based. We discussed the overall process flow of the algorithm which included the main phases of IP, feature extraction as well as the implementation and testing of the classification models. The IP entailed the contrast adjustments and the extraction of the ROI. The feature set chosen for the purpose of an overall binary classification included various levels of analysis such as global (area and volumetric ratios), regional (detection of abnormal air or masses), and local processing (texture 
analysis). The algorithm then attempted a 2-tier classification, with the primary focus on the first tier of classification, i.e., the texture analysis. A detailed discussion of the algorithm outlined the additional work and modifications required to continue the work intended for this thesis such as limiting the ROI from the entire lung to merely consider the nodule present in the lung. 


\section{4 \\ Enhanced Regression: 2D to 3D Features}

\subsection{Introduction}

While Chapter 3 discussed the construction of a feature set suitable for the task of a binary classification of lung matter, this chapter will investigate the mechanics of obtaining an enhanced feature set for the purpose of this thesis, i.e., to predict the survival time of a patient given a diagnosis of pulmonary adenocarcinoma. We will first generate a benchmark model by utilizing the same features from the prior algorithm, namely one that is pertinent to regression models. Based on the results obtained for the latter, we will discuss feature set modifications and additional feature calculations so as to improve the overall accuracy of the baseline regression models.

The main considerations that we will encounter in this chapter, will be whether new features can be calculated and appended to the existing feature set, with the goal of improving the performance when it concerns regression with respect to survival 
rates. It is important to note, however, that an increase in feature dimensionality may not necessarily be helpful in achieving better regression results. Therefore, it is mandatory for us to consider what types of features these must be, as well as their capability in capturing valuable information that is useful for regression as opposed to classification.

\subsection{Generating a Benchmark}

To adequately analyze the work done for this research, we first created a benchmark of prediction results based on the feature set used in the texture analysis phase of the prior algorithm. As described in Section 3.2.4, this feature set consisted of the Haralick values (whose formulae are defined in Appendix A) computed from the Mahotas Python library, i.e., a 12-dimensional vector.

The Haralick features were computed for every tumour patch in an image, and will henceforth be referred to as the "2D Benchmark" feature set, because the entire nodule, in its three-dimensional entirety, was not taken into consideration. For instance, if a nodule in a scan ranged over 10 images or slices, each image was treated as a single observation or record, and amounted to 10 records in the dataset. Over 60 scans, we thus extracted approximately 475 images that had a nodule present. The target variable in the data was the survival time of the patient, measured in months. The prediction of the target variable was primarily attempted with multiple baseline regression models.

\subsubsection{Model Evaluation}

As is well known for the process flow of an ML application, models need to be assessed with metrics that are relevant to the context at hand. Two measures were used to evaluate the performance of the regression models tested: The Mean Absolute Error $(M A E)$ and the Mean Relative Error (MRE), both of which are defined in Eq. (4.1) and (4.2) respectively:

$$
M A E=\frac{1}{n} \sum_{i=1}^{n}\left|y_{i}-z_{i}\right| \text {, and }
$$




$$
M R E=\frac{1}{n} \sum_{i=1}^{n} \frac{\left|y_{i}-z_{i}\right|}{z_{i}}
$$

where

- $n$ is the number of test-set data points,

- $y_{i}$ is the predicted value (i.e., the expected survival time in months),

- $z_{i}$ is the true value (i.e., the survival time in months).

The MAE is the average difference between the true value and the predicted value. It provides an overall measure of the distance between the two values, i.e., the average difference of months, but does not indicate the direction of the data (i.e., whether it is an under or over prediction). This is also known as a scale-dependent measure, as the computed values are heavily dependent on the scale of the data, and can be influenced by outliers present in the data [10]. In order to circumvent the scale-dependency, we also compute the MRE which introduces a relativity factor by dividing the error with the true value. This means that the MRE should, generally, consist of values in the range $[0,1]$. It is possible to have large or undefined values with the MRE measure when the target variable is equal to or approaches zero. However, this will not be an issue with the data used in this work as the target variable consists of non-zero numbers, since all the patients have a survival rate of at least one month.

\subsubsection{Baseline Results}

We used the scikit-learn ML library to implement the basic models for regression on the 2D Benchmark feature set. For all the testing that was done for the regression models, we maintained a consistent data split of $70 \%$ for the training and $30 \%$ for the testing. It is important to note that when analyzing the recorded metrics, we were attempting to minimize the error and maximize the accuracy of all the tested models.

As can be seen by the MRE measure, the error seems to be relatively high when compared to the results of the binary classification attained by this feature set (discussed in Section 3.2.5). Although such a performance may be undesirable, it is certainly not surprising when we take the following explanations into consideration: 
Table 4.1: Performance of Regression Models on the 2D Benchmark Dataset

\begin{tabular}{c|cc}
\hline \hline Model & MAE (months) & MRE (\%) \\
\hline Linear Regression & 12.30 & 0.76 \\
kNN Regression & 12.13 & 0.79 \\
Gradient Boosting & 12.49 & 0.77 \\
\hline
\end{tabular}

- Difference of context: This feature set was initially compiled with the goal of a binary classification between "Normal" and "Abnormal" lung texture. Processing the entire region belonging to the lung parenchyma enables the visibility of stark differences in texture which can correspond to one of the two aforementioned classes.

- Patch size: The prior algorithm processed a fixed patch size of $37 \times 37$ pixels, whereas, for this work we processed varying patch sizes depending on the size of the bounding box of the tumour in a $2 \mathrm{D}$ image.

- Tumour isolation: We considered only the tumour and not the texture around it in the 3D matrix compilation of the nodule. This means that any pixels that were included in the bounding box but were not a part of the tumour were reduced to zero (i.e., black) and ignored from feature computations. Hence, this could have had an effect on the variability seen in different tumours.

- Correlation of the features and survival time: The depth of the image with regards to the tumour would yield different feature measurements, but all slices throughout the tumour would correspond to the same value for the target value since it belonged to the same scan. This would seemingly reduce the correlation between the features and the target variable. This can be validated by Figure 4.1, which shows the correlation matrix of the feature set whereby the correlations of all the Haralick features, named Haralick0 - Haralick12, with the associated SurvivalTime, are around 0. 


\begin{tabular}{|c|c|c|c|c|c|c|c|c|c|c|c|c|c|c|c|}
\hline 1 & 0.59 & 0.72 & 0.42 & 0.22 & -0.4 & -0.22 & -0.0059 & -0.11 & -0.68 & -0.53 & -0.52 & -0.54 & -0.88 & -0.86 & - HaralickO \\
\hline 0.59 & 1 & 0.94 & 0.71 & 0.87 & 0.015 & -0.24 & 0.077 & -0.41 & -0.91 & -0.66 & -0.54 & $-0.5 ?$ & -0.64 & -0.38 & - Heralick4 \\
\hline 0.72 & 0.94 & 1 & 0.57 & 0.73 & -0.073 & -0.25 & 0.07 & -0.41 & -0.96 & -0.75 & -0.64 & -0.62 & -0.75 & -0.53 & - Haralick9 \\
\hline 0.42 & 0.71 & 0.57 & 1 & 0.63 & 0.2 & 0.11 & 0.032 & 0.12 & 0.61 & 0.43 & -0.42 & 0.43 & 0.56 & 0.32 & Heralick5 \\
\hline 0.22 & 0.82 & 0.73 & 0.63 & 1 & 0.05 & -0.15 & 0.097 & -0.36 & -0.72 & -0.69 & -0.45 & -0.42 & -0.38 & 0.023 & - Haralick11 \\
\hline-0.4 & 0.015 & -0.073 & -0.2 & 0.05 & 1 & 0.079 & -0.019 & -0.57 & 0.039 & 0.2 & 0.25 & 0.36 & 0.53 & 0.56 & - Haralick2 \\
\hline-0.22 & -0.24 & -0.25 & -0.11 & -0.15 & 0.079 & 1 & 0.023 & 0.0091 & 0.18 & 0.19 & 0.12 & 0.13 & 0.18 & 0.16 & - SurvivalTime \\
\hline-0.0059 & 0.077 & 0.07 & 0.032 & 0.097 & -0.019 & 0.023 & 1 & 0.032 & -0.044 & -0.078 & -0.013 & -0.019 & -0.0097 & 0.02 & - TumourDepth \\
\hline-0.11 & -0.41 & -0.41 & -0.12 & -0.36 & -0.51 & 0.0091 & 0.032 & 1 & 0.5 & 0.22 & 0.49 & 0.36 & 0.13 & 0.0034 & - Heralick1 \\
\hline-0.68 & -0.91 & -0.96 & -0.61 & -0.72 & 0.039 & 0.18 & -0.044 & 0.5 & 1 & 0.7 & 0.73 & 0.7 & 0.76 & 0.52 & - Haralick10 \\
\hline-0.53 & -0.66 & -0.75 & -0.43 & -0.69 & 0.2 & 0.19 & -0.078 & 0.22 & 0.7 & 1 & 0.44 & 0.44 & 0.61 & 0.39 & - Haralick12 \\
\hline-0.52 & -0.54 & -0.64 & -0.42 & -0.45 & 0.75 & 0.12 & -0.013 & 0.49 & 0.73 & 0.44 & 1 & 0.99 & 0.72 & 0.58 & - Haralick3 \\
\hline-0.54 & -0.52 & -0.62 & -0.43 & -0.42 & 0.36 & 0.13 & -0.019 & 0.36 & 0.7 & 0.44 & 0.99 & 1 & 0.75 & 0.61 & - Haralickб́ \\
\hline 0.88 & 0.64 & 0.75 & 0.56 & 0.38 & 0.53 & 0.18 & 0.0097 & 0.13 & 0.76 & 0.61 & 0.72 & 0.75 & 1 & 0.91 & Haralick7 \\
\hline-0.86 & -0.38 & -0.53 & -0.32 & 0.023 & 0.56 & 0.16 & 0.02 & 0.0034 & 0.52 & 0.39 & 0.58 & 0.61 & 0.91 & 1 & - Heralick8 \\
\hline 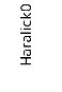 & 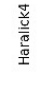 & $\begin{array}{l}\frac{0}{\vec{v}} \\
\frac{\underline{\underline{w}}}{\underline{\underline{T}}}\end{array}$ & 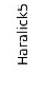 & 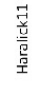 & 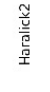 & 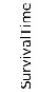 & 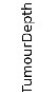 & 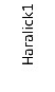 & 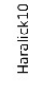 & 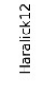 & $\begin{array}{l}\frac{m}{\underline{\underline{v}}} \\
\frac{\vec{w}}{\underline{\underline{T}}}\end{array}$ & 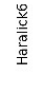 & 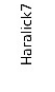 & $\begin{array}{l}\frac{\infty}{\underline{\underline{v}}} \\
\text { 몸 }\end{array}$ & \\
\hline
\end{tabular}

Figure 4.1: Correlation heatmap of the 2D Benchmark feature set.

\subsection{Benchmark Feature Set Modification}

\subsubsection{Shape Feature Calculation}

To further enhance this study, we modified the benchmark feature set by computing additional features relevant to the shape of the tumour, with an attempt to measure the "regularity" of the shape. This included calculating the following:

- Area of the tumour, measured in pixels, from the tumour mask as shown in Figure 4.2a;

- Width and height of the smallest bounding rectangle of the tumour;

- Mean Squared Error (with respect to the radius of the minimum enclosing circle of the tumour, as seen in Figure 4.2b);

- Moment values of the vector, formed by calculating the distance of the boundary of the tumour from the centre in $10^{\circ}$ increments for a full $360^{\circ}$ (as shown in 


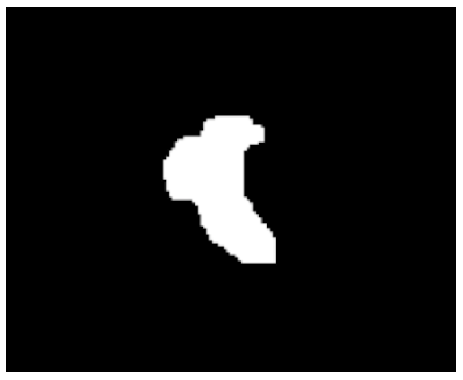

(a)

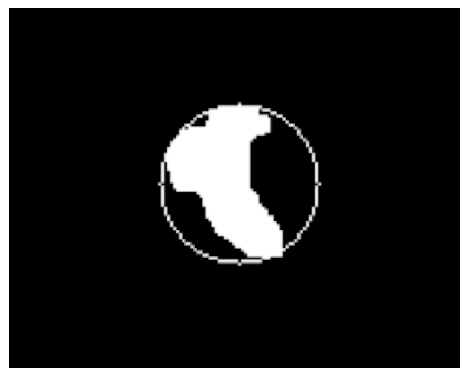

(b)

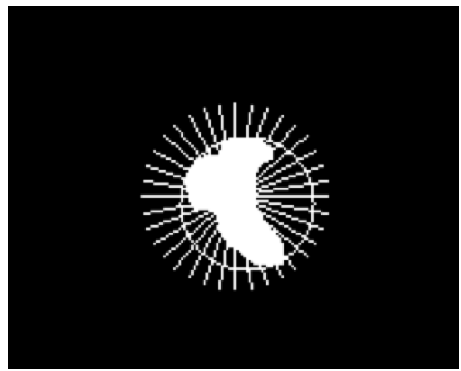

(c)

Figure 4.2: Tumour shape feature calculation process.

Figure 4.2c), where $k$ defines the $k^{\text {th }}$ central moment in Eq. (4.3).

$$
m_{k}=\frac{1}{n} \sum_{i=1}^{n}\left(x_{i}-\bar{x}\right)^{k}, \text { with }
$$

- Variance $\left(\sigma^{2}\right)$, where $k=2$, and

$$
\sigma^{2}=m_{2}
$$

- Skewness $(S)$, where $k=3$ and $s$ is the standard deviation, and

$$
S=\frac{m_{3}}{s^{3}}
$$

- Kurtosis $(K)$, where $k=4$ and $s$ is the standard deviation, and

$$
K=\frac{m_{4}}{s^{4}}
$$

This feature set will be referred to as "2D Shape v1.0" feature set. Running the same regression models as done for the baseline results, with the benchmark feature set and the augmented new shape features, yielded the error values listed in Table 4.2. As can be seen, the Linear Regression model improved in both the MAE and MRE measures. While the kNN Regression digressed with a larger error, the Gradient Boosting significantly performed much better with an improvement of $23 \%$. 
Table 4.2: Performance of Regression Models with the Shape Features.

\begin{tabular}{c|cc}
\hline \hline Model & MAE & MRE \\
\hline Linear Regression & 12.13 & 0.70 \\
kNN Regression & 14.48 & 0.89 \\
Gradient Boosting & 8.82 & 0.54 \\
\hline
\end{tabular}

\subsubsection{Shape-Based Feature Analysis}

To further analyze the new shape features, we considered the respective values against the tumour depth. Naturally, a tumour will start small and increase in size as it approaches the centre of the nodule and then decrease in size again.

\section{"Area" Feature}

We placed the shape features, beginning with "Area" $\left(a_{i}\right)$, against the "TumourDepth" $\left(t d_{i}\right)$, to observe the trend of the tumour size's progression, as visualized in Figure 4.3. We wanted to determine whether the average area and depth of the nodule had a correlation with the target variable, SurvivalTime. We then replaced the "Area" feature with the computed "MeanArea" (MA) feature, defined in Eq. (4.7), and tested the regression models. This feature set will be referred to as "2D Shape v2.0".

$$
M A=\sum_{i=1}^{i=n}\left(t d_{i} \times \frac{a_{i}}{\sum_{i=1}^{i=n} a_{i}}\right) .
$$

Table 4.3: Performance of Regression Models with Averaged "Area" Feature

\begin{tabular}{c|cc}
\hline \hline Model & MAE & MRE \\
\hline Linear Regression & 11.95 & 0.70 \\
kNN Regression & 14.52 & 0.86 \\
Gradient Boosting & 7.40 & 0.45 \\
\hline
\end{tabular}

Linear regression had a minor improvement in the MAE, although the MRE remained unchanged, indicating the same performance without taking into account the averaged "Area" feature. On the other hand, the kNN regression digressed for the 


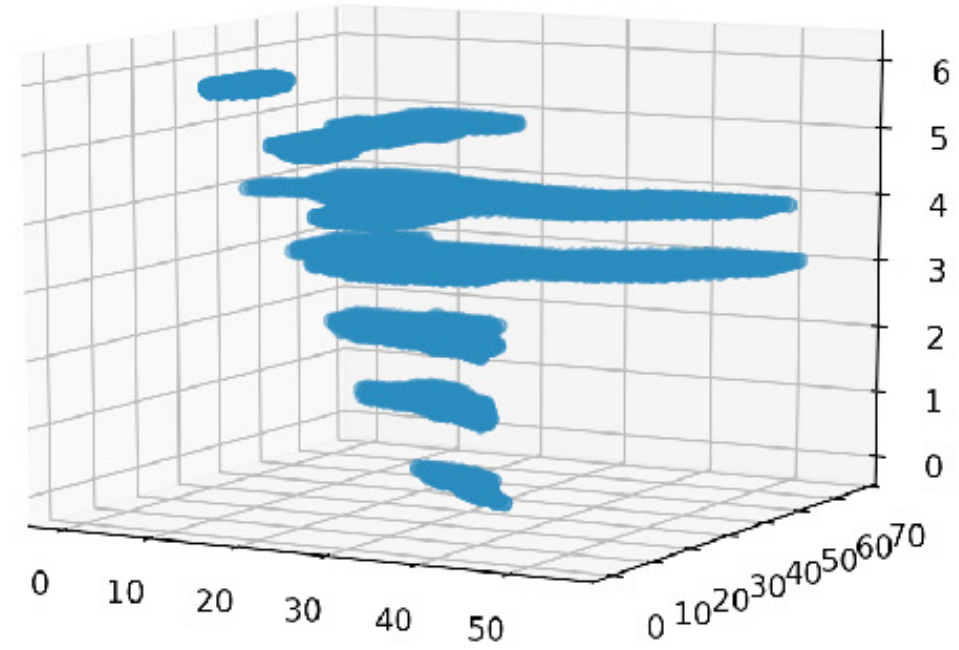

Figure 4.3: A visualization of masks of tumours from 2D images as the nodule progresses throughout the thoracic region.

MAE measure but improved by $3 \%$ for the MRE. Gradient boosting, however, had the best improvement with an approximately 15\% decrease in both the MAE and MRE. This, in and of itself, provided the basis to explore the compilation of a feature set while considering the nodule in its 3-dimensional entirety.

To enhance this further, we analyzed the averaged "Area" feature by plotting the curves of the tumour's area in relation to the depth of the image in the tumour, in 6-month bins of "SurvivalTime". The results can be seen in Figure 4.4, where the area is plotted against the percentage of tumour depth, for each 6-month bin present in the data. This was to try to understand the point in the tumour's progression where we reached the peak surface area of a 2D tumour mask in the group of scans binned for the 6 months. A notable observation was the steady trend of decreasing "MeanArea" over the first four bins (i.e., those that had a survival time within 2 years of diagnosis).

Given this trend, we also tested the regression models on a subset of the data which had a "SurvivalTime" of 24 months or less. Subsets were taken of all of the aforementioned feature sets, and processed to recalculate the errors. Table 4.4 displays the results of the regression models with respect to each feature set. 


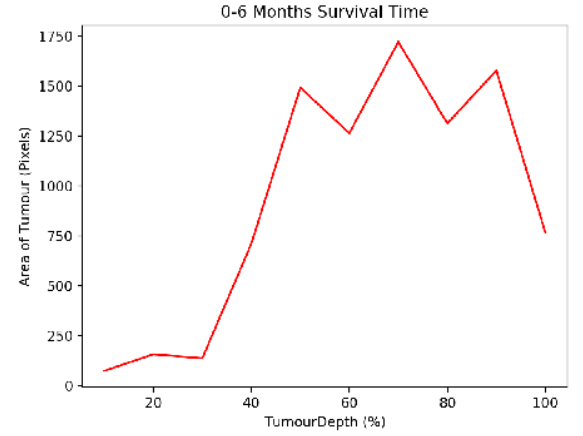

(a) Mean $=0.685$

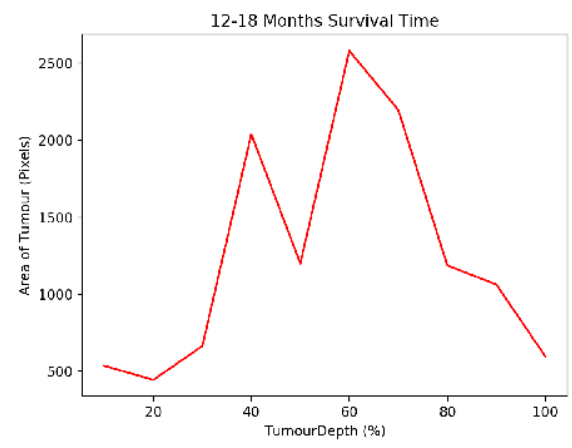

(c) Mean $=0.579$

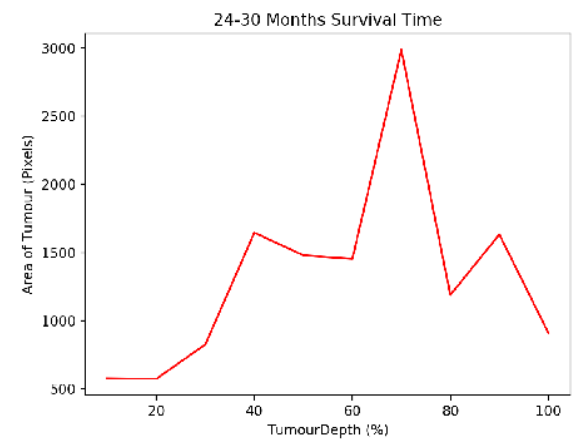

(e) Mean $=0.589$

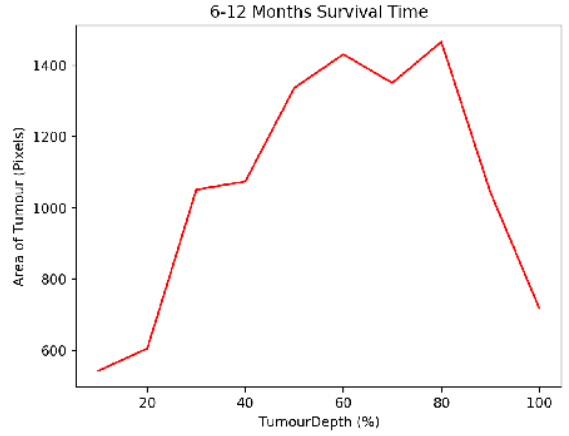

(b) Mean $=0.591$

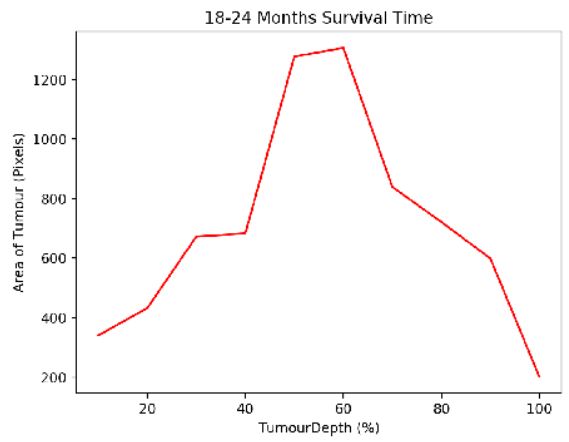

(d) Mean $=0.555$

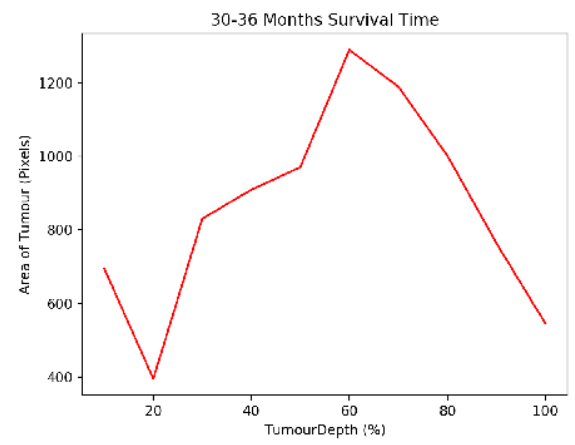

(f) Mean $=0.566$

Figure 4.4: Plots of the Mean of the Area based on the tumour's progression for binned survival times between 0 months and 36 months. 


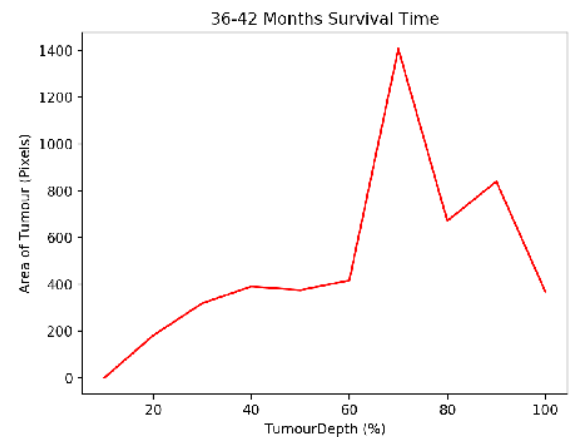

(g) Mean $=0.679$

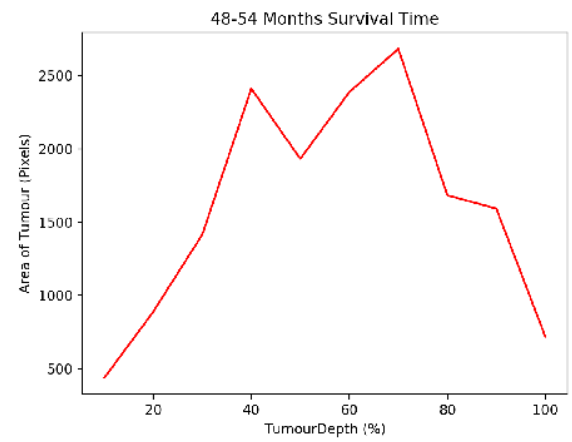

(i) Mean $=0.585$

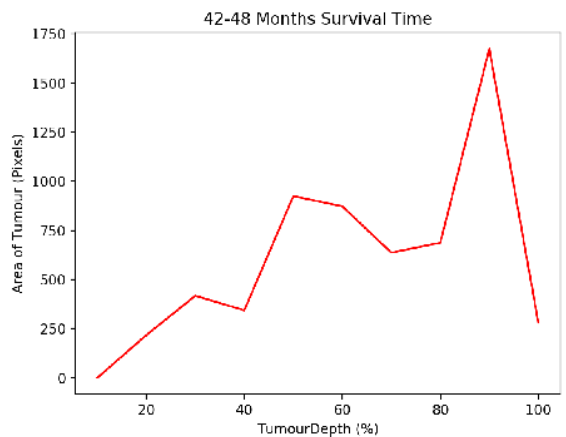

(h) Mean $=0.671$

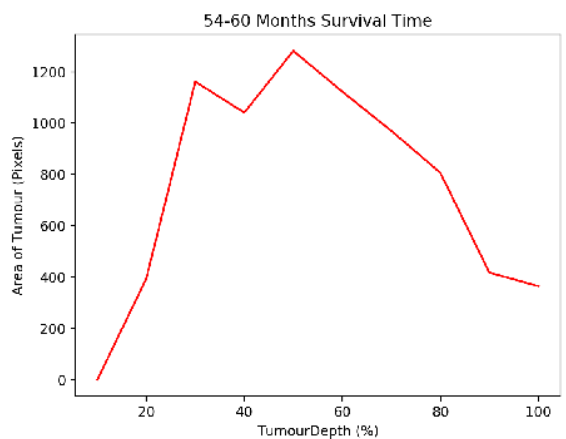

(j) Mean $=0.546$

Figure 4.4: Plots of the Mean of the Area based on the tumour's progression for binned survival times between 36 months and 60 months.

Table 4.4: Regression Results with Data Subset: "SurvivalTime" Less Than 24 Months.

\begin{tabular}{l|l|ccc}
\hline \hline \multirow{2}{*}{ Feature Set } & Evaluation & \multicolumn{3}{|c}{ Regression Model } \\
& Metric & Linear & kNN & Gradient Boosting \\
\hline \multirow{2}{*}{ 2D Benchmark } & MAE & 4.27 & 5.22 & 4.56 \\
& MRE & 0.35 & 0.40 & 0.37 \\
\hline \multirow{2}{*}{ 2D Shape v1.0 } & MAE & 3.53 & 4.60 & 1.79 \\
& MRE & 0.27 & 0.35 & 0.12 \\
\hline \multirow{2}{*}{ 2D Shape v2.0 } & MAE & 3.23 & 5.33 & 1.24 \\
& MRE & 0.26 & 0.41 & 0.09 \\
\hline
\end{tabular}




\section{"DistanceSkewness" Feature}

A similar analysis was conducted for one of the distance moment features, "DistanceSkewness", as the moment features differ only by the order of magnitude. This feature set will be referred to as "2D Shape v2.1" as it will only include the newly computed "DistanceSkewness" averages, and not the "Area" averages in order to observe the direct result of the averaging. The results are shown in Table 4.5, where it can be seen that there was a minor improvement of $8 \%$ and $6 \%$ in the MRE for $\mathrm{kNN}$ regression and Gradient Boosting respectively, when compared to the results shown in Table 4.2. Clearly, this still supported the basis for compilation of a 3D feature set.

Table 4.5: Performance of Regression Models with Averaged "DistanceSkewness" Feature (2D Shape v2.1).

\begin{tabular}{c|cc}
\hline \hline Model & MAE & MRE \\
\hline Linear Regression & 12.29 & 0.71 \\
kNN Regression & 13.55 & 0.81 \\
Gradient Boosting & 7.53 & 0.46 \\
\hline
\end{tabular}

Table 4.6 displays the average of this feature, for every 6-month bin, to consider any possible short-term trend. As can be seen, there is no evident trend for the average as the "SurvivalTime" of a patient increases. Despite this lack of a significant trend, we can see from Table 4.7 that the feature set "2D Shape v2.1" still performed significantly better on all regression models.

\subsection{Chapter Conclusions}

In this chapter, we first tested the same feature set that was used in the prior algorithm, to consider how it would perform for regression models. However, due to multiple reasons such as a difference of context, narrowed focus on the cancer nodule, as well as processing differences, the regression results yielded large error measures when compared against the accuracy of the original binary classification. 
Table 4.6: Averages of "DistanceSkewness" over 6-Month Bins.

\begin{tabular}{c|c}
\hline \hline "SurvivalTime" Bin & Average \\
\hline$(0,6]$ & -1.74 \\
$(6,12]$ & 0.47 \\
$(12,18]$ & 1.40 \\
$(18,24]$ & 1.18 \\
$(24,30]$ & 0.31 \\
$(30,36]$ & 0.08 \\
$(36,42]$ & 0.33 \\
$(42,48]$ & 1.85 \\
$(48,54]$ & 0.16 \\
$(54,60]$ & 0.00 \\
\hline
\end{tabular}

Table 4.7: Performance of Regression Models with Feature Set 2D Shape v2.1 and "SurvivalTime" Less Than 24 Months.

\begin{tabular}{c|cc}
\hline \hline Model & MAE & MRE \\
\hline Linear Regression & 3.28 & 0.27 \\
kNN Regression & 4.60 & 0.35 \\
Gradient Boosting & 1.23 & 0.09 \\
\hline
\end{tabular}


Modifying the existing feature set with the addition of shape descriptors, which were more focused on the cancer nodule itself, showed improvements in some of the regression models. These new features were then analyzed against the "TumourDepth", from which a strong correlation between the images at successive layers of the scan was found. Further investigating the progression of the "Area" feature values versus tumour progression in bins of "SurvivalTime" displayed a notable trend for survival rates up to 24 months. The regression testing for the dataset containing these patients achieved an MRE as low as $9 \%$ and an improvement of up to $36 \%$ for the Gradient Boosting scheme. 


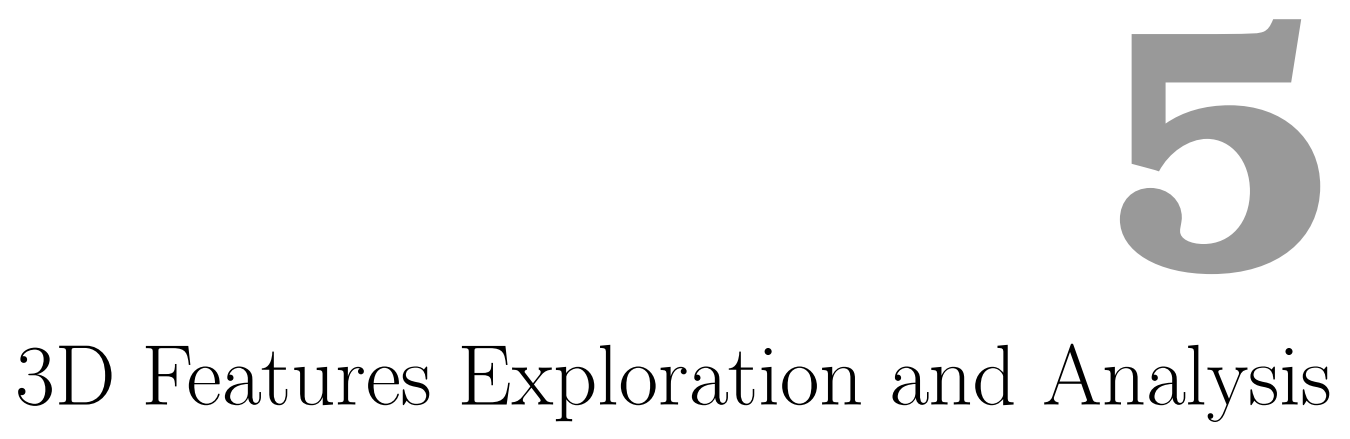

\section{$5.1 \quad$ Introduction}

The problems that we have been considering until now have basically dealt with generalizing classification-based results into the regression domain using features that were used earlier or enhanced in 2- and 3-dimensions. The interesting and significant contribution for the 3-dimensional enhancement was that we were able to demonstrate that the shape of the tumour, as it proceeded down the thoracic region, had a strong correlation with the survival rate. This was previously an unreported fact, that helped us to achieve far superior regression results.

In this chapter, we shall attempt to take "another leap of faith". The premise for the research here is that we can find many more 3D features which can be used to augment the feature vector to the previously-computed 3D features. The dilemma that we face is the following: Increasing the dimensionality of the feature vector need 
not necessarily increase the accuracy of a regression scheme. While such a strategy is beneficial in the case of classification, it will definitely not be expedient for regression, especially because the scales of the different features could skew the corresponding results. So the question that we now face is the following: Given a largely-enhanced feature vector, is it possible to reduce the dimensionality of this vector so as to obtain superior regression results?

This chapter will firstly consider augmenting the 3D features used in Chapter 4 to create a new feature set that has more than 100 features. Obviously, working with such a large feature set is both time-consuming and cumbersome. Our main hypothesis is that we can reduce the dimensionality of this feature vector to get finer regression results. We shall achieve this in three ways:

- We will implement three different feature selection techniques, namely variance thresholding, recursive feature elimination, and tree-based feature selection, to get a subset of the data that has a greater association with the target variable, resulting in better performance.

- We will invoke the well-known PCA scheme and work with its reduced feature vector. The results that we obtain are noticeably superior.

- We will embark on a whitening process based on the eigenvalues and eigenvectors of this feature space. Notice that since we only have a single class (as opposed to multiple classes), the diagonalization will be in the directions of the eigenvectors of this single class. Based on these eigenvalues and eigenvectors, we shall reduce the dimensionality of the space and then invoke the same regression models as we did in Chapter 4.

\subsection{D Feature Set Compilation}

Proceeding now with the work that we did, the next stage included the exploration, compilation and modification of a 3D feature set, which essentially implies that we considered the entire lung as a single entity (or observation) as opposed to the images considered for the 2D feature sets alluded to in the previous sections. We used the 
Pyradiomics library [40], an open source Python package, for extracting radiomics ${ }^{1}$ data from medical images.

The main emphasis for this feature set was to compute measures in a 3D consideration. Compared to the benchmark feature set, which contained quantification measures of texture analysis in the $2 \mathrm{D}$ domain, this feature set includes recalculated texture analysis components in a 26-connectivity capacity, i.e., if the centre pixel shares a face, edge or corner with another pixel. The radiomics features extracted using the Pyradiomics library are defined in sub-categories known as feature classes, listed below. The individual features calculated in each class are defined in the Pyradiomics documentation ${ }^{2}$.

- First Order Statistics: This feature class focuses on computing features that describe the histogram (defined in Section ??) of the nodule image, i.e., the grey-level intensity distribution. These measures include the mean, median, energy, entropy, uniformity, etc., amounting to a total of 19 features.

- 3D Shape-based: These measures, unlike the rest of the feature classes, compute the values from the mask of the given nodule as they are independent of the grey level intensities. The Pyradiomics library builds a triangle mesh from the provided mask using the marching cubes algorithm. The triangle mesh aids in calculating features including mesh volume, voxel volume, surface area, sphericity, compactness among many others amounting to a total of 16 features.

- Grey Level Cooccurrence Matrix (GLCM): This matrix, also discussed in Section 2.2.1, is computed based on the probability function where the $(i, j)^{\text {th }}$ element represents the number of times grey levels $i$ and $j$ appear next to each other in the image. For 3D purposes, the GLCM computation considers a 26connectivity algorithm, given that the distance from the centre voxel is unity. The GLCM is quantified by individual measures such as autocorrelation, cluster

\footnotetext{
${ }^{1}$ Radiomics is the field of study that extracts quantitative measures of tumour phenotypes from medical images using data-characterization algorithms. These features are explored to uncover disease characteristics that are not visible to the naked eye, which can then be used for further prognosis and treatment plans.

${ }^{2}$ Documentation is available at https://pyradiomics.readthedocs.io/en/latest/.
} 
aspects, entropy, variance, inverse different moment.This phase leads to a total of 24 features.

- Grey Level Run Length Matrix (GLRLM): This matrix captures grey level runs, in which, a run refers to the number of consecutive pixels that have the same grey level value. Hence, the $(i, j)^{\text {th }}$ element represents the number of runs that occur with grey level $i$ and length $j$. The GLRLM is then quantified by individual measures such as short run and long run emphasis, non-uniformity values, run variance, etc., amounting to a total of 16 features. Some of these features, briefly mentioned in 3.2.4, were initially included in the "2D Benchmark Feature Set" from the prior algorithm, but were removed through employed feature selection techniques.

- Grey Level Size Zone Matrix (GLSZM): This matrix is similar to the GLRLM, however, voxels of the same grey level intensity are taken into consideration rather than just pixels. The number of connected voxels that share the intensity form a zone, and therefore, the $(i, j)^{\text {th }}$ element represents the number of zones that occur with grey level $i$ and size $j$. The GLSZM is quantified by individual measures such as area and zone emphasis, non-uniformity, variance, entropy, etc. amounting to a total of 16 features.

- Neighbouring Grey Tone Difference Matrix (NGTDM): This matrix evaluates the difference between a pixel or voxel's grey value and the values of its neighbours. From the NGTDM, individual measures such as contrast, complexity, strength, and coarseness are calculated amounting to a total of 5 features.

- Grey Level Dependence Matrix (GLDM): This matrix measures the grey level dependencies in the image. As mentioned in the Pyradiomics documentation, a neighbouring voxel with grey level $j$ is considered dependent on the centre voxel with grey level $i$ if the difference between the two intensities is above a specified threshold. The GLDM is described with individual measures such as dependence emphases, non-uniformity variations, entropy, variance, etc. 
amounting to a total of 14 features.

\subsubsection{Evaluation of Feature Space}

Despite the exclusion of the 2D shape features, the resulting set consisted of a 110dimensional feature vector which, for a total of 60 data points, was quite excessive. We implemented feature selection and dimensionality reduction techniques to yield the most efficient, compact, and representative feature set.

First and foremost, we took the baseline 3D feature set, i.e., the set that is inclusive of all the Pyradiomics features mentioned previously, appended with the previouslycomputed 3D features from Section 4.3.2. This provided us with a criterion to evaluate the performance improvement from different feature selection techniques. Running the same regression models as discussed earlier, Table 5.1 displays the results for the feature set, referred to as "3D Baseline". The high error measures were expected given the high probability of overfitting the regression models due to the large feature space.

Table 5.1: Performance of Regression Models with "3D Baseline" Feature Set.

\begin{tabular}{c|cc}
\hline \hline Model & MAE & MRE \\
\hline Linear Regression & 34.41 & 1.95 \\
kNN Regression & 16.73 & 0.97 \\
Gradient Boosting & 14.76 & 0.85 \\
\hline
\end{tabular}

\subsection{Feature Selection Techniques}

We implemented a total of three basic feature selection techniques (listed below) for exploratory purposes, to understand which combination of the features computed from Pyradiomics would yield the best results.

1. Variance Thresholding: This strategy removes all features whose variance does not meet the specified threshold. In our implementation, we used 0.25 as our testing threshold. This resulted in a 53-dimensional feature vector, which 
was still considerably high. The feature set is referred to as "3D Reduced v2.1" in the results displayed in Table 5.2. For experimentation, we gradually increased the variance threshold in search of such a value that would limit the feature set to at most 20 features. However, increasing it up to even 0.95 resulted in a 52-dimensional feature vector.

2. Recursive Feature Elimination: Using this method, the selection of features was done recursively by examining decreasingly smaller sets of features, whereby features that were deemed as "unimportant" through the measure of a prespecified coefficient, were excluded in the next set. The function was customized to choose the top 10 features. We chose the Gradient Boosting Regressor as the model for recursive selection due to its performance when evaluating 2D datasets. This feature set is referred to as "3D Reduced v2.2".

3. Tree-based Feature Selection: In this scheme, we utilized the forest of trees, i.e., an ensemble, to compute feature importances. We customized this method to choose the 15 most important features and the feature set is referred to as "3D Reduced v2.3".

Table 5.2: 3D Feature Set Regression Results with Feature Selection

\begin{tabular}{l|l|ccc}
\hline \hline \multirow{2}{*}{ Feature Set } & Evaluation & \multicolumn{3}{|c}{ Regression Model } \\
& Metric & Linear & kNN & Gradient Boosting \\
\hline \multirow{2}{*}{ 3D Reduced v2.1 } & MAE & 74.11 & 16.73 & 16.04 \\
& MRE & 3.65 & 0.97 & 0.80 \\
\hline \multirow{2}{*}{ 3D Reduced v2.2 } & MAE & 11.77 & 14.58 & 12.48 \\
& MRE & 0.62 & 0.78 & 0.77 \\
\hline \multirow{2}{*}{ 3D Reduced v2.3 } & MAE & 21.11 & 16.03 & 15.44 \\
& MRE & 1.02 & 0.76 & 0.83 \\
\hline
\end{tabular}

Considering Table 5.2, although the performance of Linear Regression worsened by more than two times for the feature set generated from variance thresholding, the best performance was also achieved from Linear Regression, with the feature set attained from recursive feature elimination obtaining an MAE of 11.77 and MRE of 
0.62. This was also an improvement when compared to the performance of Linear Regression on the 2D datasets where we considered the entire dataset.

\subsubsection{Short-term Prediction Results}

To maintain consistency between 2D and 3D feature set analyses, we ran the regression models on all versions of the 3D feature sets with the subset of the data which has a SurvivalTime of less than or equal to 24 months.

Table 5.3: 3D Feature Set Regression Results with SurvivalTime $\leq 24$ Months

\begin{tabular}{l|l|ccc}
\hline \hline \multirow{2}{*}{ Feature Set } & Evaluation & \multicolumn{3}{|c}{ Regression Model } \\
3D Baseline & Metric & Linear & kNN & Gradient Boosting \\
\hline \multirow{2}{*}{ 3D Reduced v2.1 } & MAE & 58.05 & 5.8 & 7.07 \\
& MRE & 5.61 & 0.41 & 0.59 \\
\hline \multirow{2}{*}{ 3D Reduced v2.2 } & MRE & 57.94 & 5.8 & 7.18 \\
& MAE & 5.60 & 0.41 & 0.53 \\
\hline \multirow{2}{*}{ 3D Reduced v2.3 } & MRE & 0.76 & 0.24 & 7.38 \\
& MRE & 16.42 & 7.32 & 0.65 \\
\hline
\end{tabular}

The best performance was achieved with kNN regression on the feature set generated with recursive feature elimination with an MAE of 4.36, and an MRE of only $24 \%$.

\subsection{Dimensionality Reduction Techniques}

For further considerations in the analysis of obtaining an informative 3D feature set, we also implemented dimensionality reduction techniques. While both feature selection and dimensionality reduction techniques aim to reduce the number of attributes in a dataset, the former will return a subset of the original data, while the latter reduced the data by creating new combinations of the attributes. For dimensionality reduction, we first considered the well-known Principal Component Analysis (PCA) technique. 


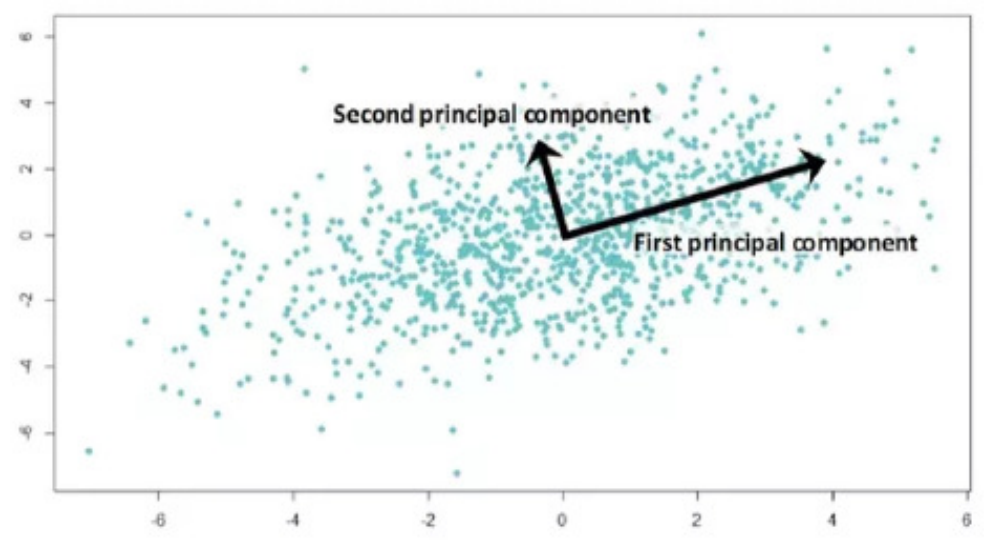

Figure 5.1: A visualization of PCA.

\subsubsection{Principal Component Analysis (PCA)}

Principal Component Analysis is the process whereby a data set is converted using orthogonal transformations to build a data set of linearly uncorrelated variables known as the set's principal components. It can be considered as fitting a $p$-dimensional ellipsoid to the data at hand. Every axis of the new ellipsoid is a principal component. The larger the axis, the more variance that is captured in that component. This is achieved in such a way that the first component captures the largest variance (i.e., the most information) in the dataset, and each consecutive component will have the next highest variance. Consider Figure 5.1 as a simplistic visual explanation of the PCA in comparison to feature variance. This diagram shows a plot of a 2-dimensional dataset, while real-world datasets will obviously have many more, where if a 2-dimensional ellipsoid were to be fit around it, the major axis would be in the direction of the arrow labeled first principal component due to the larger variance.

Before invoking PCA, the dataset had to be standardized so as to remove the bias of variances in the initial variables. After standardization, we implemented the PCA using the Python framework scikit-learn for both scaling the data and fitting the PCA transformation. We limited the transformation to the creation of 5 new features, for which the results are displayed in Table 5.4.

The explained variation for the principal components for the five components is 
Table 5.4: Performance of Regression Models with Feature Set "3D Reduced v3.0".

\begin{tabular}{c|cc}
\hline \hline Model & MAE & MRE \\
\hline Linear Regression & 14.83 & 0.78 \\
kNN Regression & 17.15 & 0.90 \\
Gradient Boosting & 18.36 & 1.00 \\
\hline
\end{tabular}

as follows: $[0.476,0.165,0.092,0.055,0.042]$. As can be seen, the first two components contain the most significant information, and so the regression models were re-tested to obtain the PCA results with the creation of only 2 new features (referred to as "3D Reduced v3.1").

Table 5.5: Performance of Regression Models with Feature Set "3D Reduced v3.1”.

\begin{tabular}{c|cc}
\hline \hline Model & MAE & MRE \\
\hline Linear Regression & 14.72 & 0.81 \\
kNN Regression & 17.30 & 1.01 \\
Gradient Boosting & 15.33 & 0.85 \\
\hline
\end{tabular}

While we observed a minor degradation in performance for Linear and kNN Regression models, displayed in Table 5.5, the Gradient Boosting regression model improved significantly with a $15 \%$ improvement in the MRE when we considered only the first two principal components. However, there were negligible differences when compared to the baseline results of this dataset (refer to Table 5.1) for both the kNN regression and gradient boosting, with the former facing a degradation of $3 \%$. Linear regression, however, had a significant improvement of over $100 \%$.

\subsection{Data Diagonalization}

The fundamental principle motivating dimensionality reduction involves processing the covariance matrix of the data and reducing the dimensions based on the principal, or rather, significant eigenvalues. This is also the motivation for the PCA method described in the previous section. In this section, we shall show how we can use a non-traditional eigenvector matrix to further enhance the accuracy of the scheme, and also simultaneously minimize the computations. 


$$
\left[\begin{array}{cccccc}
B_{1} & 0 & 0 & 0 & 0 \\
\cline { 1 - 1 } 0 & B_{2} & 0 & 0 & 0 \\
0 & 0 & B_{3} & 0 & 0 \\
0 & 0 & 0 & B_{4} & 0 \\
0 & 0 & 0 & 0 & B_{5}
\end{array}\right]
$$

Figure 5.2: Block diagonal matrix.

The monumental task associated with a covariance matrix of large dimensions is to obtain its eigenvalues and eigenvectors. While this is surely advantageous, the task is daunting especially when we deal with a feature space whose dimensionality is greater than 100. It would have been meaningful if we were working with simultaneous diagonalization where we tried to diagonalize or whiten data from two classes. In our case, however, since the problem we tackle is regression (rather than classification), we are dealing with only a single class which makes the problem less cumbersome.

As mentioned in the last section, the dimensionality of the feature vector is 110 . Computing the eigenvalues and eigenvectors of such a large matrix is, certainly, time consuming. The novel contribution in this section is that we advocate dividing the feature vector into multiple sub-vectors, for example, 5 sub-vectors. We are now faced with a problem of getting 5 sets of eigenvectors, each of dimension 22, which is a significantly smaller problem. Of course, this leads us to an approximated world in which the features within these subspace are correlated, but it leads to a model in which the features outside of these blocks are uncorrelated. This, in turn, leads to the concept of a block diagonal matrix, as displayed in Figure 5.2, where the blocks, $B_{n}$ where $n=1 \ldots 5$, represents the subset of features chosen (i.e., 5 sets of 22 -dimensional vectors), and all other elements outside of the blocks are set to zero.

Our task now, is to diagonalize each of these blocks within the block diagonal approximation, and to choose a subset of their prominent eigen-directions, which are determined by the corresponding largest eigenvalues. For example, if we extracted the 5 principal eigen-directions in each of these blocks, the 110-vector space would reduce to 5 blocks of 5 features each, i.e., a feature vector of dimension 25. Regression 
is now done by getting the best value based on each of these sub-blocks and averaging between them. The reader will observe that this is essentially an ensemble machine, where the 5 blocks lead to their own respective regression values, which are then averaged to obtain the overall solution. Although this involves computations that are significantly less than working with the 110-dimensional space, the results that we have obtained are actually marginally superior. This seems to be paradoxical but the reason for this is probably because the higher dimensional world tries to impose a dependence on the various variables when, in fact, there may not be such an explicit dependence relationship.

\subsubsection{Implementation and Results}

The diagonalization technique applied to the dataset, defined in Eq. (5.1), is a linear transformation that transforms a set of vectors, $\{X\}$, with a given covariance matrix to a new set of vectors, $\{Y\}$, with a covariance that is the identity matrix. This indicates uncorrelated features with a variance of 1 satisfying:

$$
Y=W^{T} X
$$

The transformative factor here is defined by $W$, which is a $d \times d$ matrix such that:

$$
\begin{gathered}
W^{T} \Sigma_{X} W=\Lambda, \text { and } \\
\Lambda=\left[\begin{array}{lll}
\lambda_{1} & \ldots & 0 \\
& \ddots & \\
0 & \ldots & \lambda_{d}
\end{array}\right],
\end{gathered}
$$

where $\lambda_{1} \ldots \lambda_{n}$ are the eigenvalues of the covariance matrix. It is important to note that $W$ also satisfies the following condition:

$$
W^{-1}=W^{T} .
$$

As mentioned earlier, the context at hand is a regression problem and hence, we are dealing with data from only a single class. Therefore, we implement a simple diagonalization rather than a simultaneous diagonalization of multiple classes. 
We computed the covariance matrix of the scaled dataset, resulting in a $110 \times 110$ matrix. However, computing the eigenvalues and eigenvectors of the covariance matrix was computationally complex and inefficient, resulting in negligibly-small negative and complex eigenvalues ${ }^{3}$. To combat the inaccuracy of these results, we broke down the 110-dimensional covariance matrix into five 22-dimensional covariance matrices from which we could compute the eigenvalues. This led to the resemblance of an ensemble-based model, as we transformed the data five times with only the $k^{\text {th }}$ group of features extracted from the covariance matrix. Running the regression models with selecting the corresponding transformed features of the first $d$ significant eigenvalues (where $d$ is the number of eigenvalues that have values above a threshold of 1.0), we took the average of the five predictions and attained our final regressed prediction.

Table 5.6: Performance of Regression Models with Feature Set "3D Reduced v4.0".

\begin{tabular}{c|cc}
\hline \hline Model & MAE & MRE \\
\hline Linear Regression & 14.28 & 0.76 \\
kNN Regression & 15.41 & 0.83 \\
Gradient Boosting & 15.13 & 0.80 \\
\hline
\end{tabular}

Table 5.6 shows the regression results on the diagonalized data. Compared to results from PCA, there seems to be an overall improvement across all models with gradient boosting improving the most, with a 20\% decrease in the MRE. Table 5.7 displays the regression results on the subset data where the survival time was less than or equal to 24 months. As expected, all the regression models improved greatly with an average decrease in the MAE of over $50 \%$, and the MRE reaching as low as $41 \%$ with gradient boosting.

\subsection{Chapter Conclusions}

This chapter explored the engineering of a feature set based on 3D analyses and the related computations associated with the tumour. The foundation of focussing on

\footnotetext{
${ }^{3}$ This was, of course, due to the computations involving very small quantities.
} 
Table 5.7: Performance of Regression Models with Feature Set "3D Reduced v4.0" on Subset Data.

\begin{tabular}{c|cc}
\hline \hline Model & MAE & MRE \\
\hline Linear Regression & 6.98 & 0.53 \\
kNN Regression & 6.10 & 0.42 \\
Gradient Boosting & 5.89 & 0.41 \\
\hline
\end{tabular}

extracting features while taking the entire nodule into consideration, was formed in Chapter 4, where considering shape-based features against the tumour depth, drastically improved our results. This implied the presence of correlations between feature values and the progression of the tumour. We employed Pyradiomics for the computation of the relevant features to both shape and texture, in the 3D aspect. This resulted in a 110-dimensional feature vector, which we further analyzed with feature selection and dimensionality transformation techniques.

We achieved an overall improvement in performance from the baseline of the 3D feature set to an MRE of $62 \%$ with the feature set that was obtained through recursive feature elimination, which resulted in a 10-dimensional feature vector. Notably, the same feature set also performed the best with an MRE of $24 \%$ on the subset data of survival time less than or equal to 24 months.

When comparing the results found in this chapter to that of the previous chapter with the 2D features, the baseline results from Chapter 4 (i.e., Table 4.1) prove to be significantly better across all regression models than the baseline results for the 3D features (i.e., Table 5.1). However, this can easily be attributed to the vast increase in the feature space. Considering results on the entire set of data, the lowest MRE achieved by linear regression on the "3D Reduced v2.2" feature set of $62 \%$ is comparable to the lowest MRE of 54\% for 2D features (excluding the averaged "Area" feature) achieved by gradient boosting. 


\section{6 \\ Conclusion}

In this chapter, we will provide a summary of the work entailed in this thesis, the results that have been attained, and discuss the outcomes with respect to others who investigated the same dataset. Finally, we will conclude with potential avenues for future work and research.

\subsection{Summary}

The goal of this thesis was to tackle the problem of predicting survival rates of patients diagnosed with adenocarcinoma, or in other terms, to evaluate the severity of a cancer nodule. Achieving this with a high confidence interval can be a breakthrough for determining the prognosis and treatment plan of an illness. We considered chest CT scans, and by implementing image processing techniques, extracted what we believed would be informative and indicative features with regards to the survival time, i.e., 
the target variable. We examined both 2D images in and of themselves, as well as the 3D nodule in its entirety, when creating feature sets.

In Chapter 2, we surveyed the fields of medical imaging, and applications of both, IP and ML in healthcare and diagnostics. We reviewed ML for images, the types of operations for feature extraction and the suitable classifiers for different image classification contexts. The chapter then reviewed the background of medical imaging and measurements, especially those attained from CT scanners, such as Hounsfield Units, and contrast windowing concepts. Lastly, but most importantly, the survey discussed the existing literature for IP and ML applications in the context of medical image reports, including but not limited to the preferred preprocessing techniques, nodule detection, segmentation, and classification.

In Chapter 3, we described the foundational algorithm which formed the basis of this thesis, which was essentially a CAD prototype with a 2-tier classification for an overall binary classification. We reviewed the IP techniques employed for the preprocessing (filtering, contrast adjustment, and lung segmentation), the feature extraction process (local and global image processing, computation of area and volumetric ratios, and texture analysis), as well as the tested classification methods in both tiers of the system. We also discussed the additional work of nodule segmentation, to ensure the inclusion of juxtapleural nodules and the reduction of the computational space, as a pre-requisite for the work for this thesis.

In Chapter 4, we focused on 2D images of the $\mathrm{CT}$ scans to maintain the corresponding processing infrastructure of the previously-mentioned algorithm. We constructed a benchmark by testing regression models on the texture-based feature set from the foundational algorithm. These results, as expected, were less accurate than the results obtained from the binary classification due to a number of reasons such as a difference of context in terms of the target variable, the isolation of the tumour for processing, and the low correlation values of the features and survival time. We appended this set with shape-descriptor features, which enhanced the regression results, although not significantly. We then proposed considering the features in the successive sequence of images, with regards to the depth of the tumour, and appended the feature set with an averaged feature for each feature evaluated. This was a notable breakthrough, as 
our accuracy doubled and provided the basis to explore 3D features.

In Chapter 5, we explored the construction of a feature set based on 3D computations. Using the Pyradiomics Python library, we obtained a 110-dimensional feature vector. With a regression problem at hand, an excessive number of features was not necessarily beneficial, and hence, the major focus for this chapter was to reduce the dimensionality. We tested this with both feature selection as well as dimensionality transformative techniques. We observed a substantial improvement in regression results with all techniques when compared to the baseline, and more specifically, recursive feature elimination achieved the greatest improvement in accuracy of over $100 \%$. The diagonalization of the data also performed better than the wellknown PCA scheme by $20 \%$. Notably, we were also able to reduce the computational complexity for eigenvalues and eigenvectors through a block-diagonal scheme.

\subsection{Conclusion and Achievements}

To conclude this thesis, we briefly state our key findings and significant achievements:

- We performed sequential processing by aggregating $2 \mathrm{D}$ features through their measurements, in successive images of the scan, and included it as a single representative feature. This achieved a performance enhancement of almost $30 \%$.

- We "binned" the scans according to their survival times and executed an indepth analysis of the "Averaged Area" feature shape descriptor against the progression of the tumour. We were thus, able to determine that the regression analysis generally performed far more accurately when dealing with short term predictions, as opposed to long term predictions. In fact, in some cases, we attained error measures as low as $9 \%$.

- We invoked a novel block-diagonal scheme for feature reduction. This was done so as to design a new (or rather, previously unreported) data diagonalizationbased feature reduction technique. This novel scheme attained more superior 
results of approximately $20 \%$ improvement when compared to the transformed feature set acquired through a PCA-based implementation.

\subsection{Future Work}

Lastly, we propose future avenues of research, including but not limited to:

- Exploring a wider range of regression models with more effort on parameter tuning of the models.

- Exploring more texture-based features, perhaps by analyzing feature computations from successive 2D images as opposed to a direct 3D computations to achieve an even higher accuracy, as seen in Chapter 4 .

- Testing the explored features with another dataset of chest CT scans with adenocarcinoma to ensure the consistency of the results.

- With the notable findings that we have obtained, we note that there are an abundance of other pathologies that encompass similar characteristics, where a study on the severity that is not visible with the naked eye, could aid in decisions pertaining to potential treatment plans. 


\section{Bibliography}

[1] B. Al Mohammad, P. C. Brennan, and C. Mello-Thoms. A review of lung cancer screening and the role of computer-aided detection, 2017.

[2] S. G. Armato III and W. F. Sensakovic. Automated lung segmentation for thoracic ct: Impact on computer-aided diagnosis. Academic Radiology, 11:10111021,2004

[3] S. G. Armato III and W. F. Sensakovic. Automated lung segmentation for thoracic ct: Impact on computer-aided diagnosis. Academic Radiology, 11:10111021, 2004.

[4] S. G. Armato III, M. L. Giger, and H. MacMahon. Automated detection of lung nodules in ct scans: Preliminary results. Medical Physics, 28:1552-1561, 2001.

[5] M. S. Bartlett, G. Littlewort, M. Frank, C. Lainscesk, I. Fasel, and J. Movellan. Recognizing facial expression: Machine learning and application to spontaneous behaviour. Computer Vision and Pattern Recognition, 2:568-573, 2005.

[6] H. Bay, T. Tuytelaars, and L. V. Gool. Surf: Speeded up robust features. European Conference on Computer Vision, pages 404-417, 2006.

[7] B. Caldairou, N. Passat, P. A. Habas, C. Studholme, and F. Rousseau. A nonlocal fuzzy segmentation method: Application to brain mri. Pattern Recognition, 44:1916-1927, 2011. 
[8] F. Chabat, G. Z. Yang, and D. M. Hansell. Obstructive lung diseases: Texture classification for differentiation at ct. Radiology, 228:871-877, 2003.

[9] O. Chapelle, P. Haffner, and V. N. Vapnick. Support vector machines for histogram-based image classification. IEEE Transactions on Neural Networks, 10:1055-1064, 1999.

[10] C. Chen, J. Twycross, and J. M. Garibaldi. A new accuracy measure based on bounded relative error for time series forecasting. PloS one, 12, 2017.

[11] W. Cheung and G. Hamarneh. n-sift: n-dimensional scale invariant feature transform. Biomedical Imaging: From Nano to Macro, pages 2012-2021, 2009.

[12] R. S. Choras. Image feature extraction techniques and their applications for cbir and biometric systems. International Journal of Biology and Biomedical Engineering, 2007.

[13] D. C. Ciresan, U. Meier, J. Masci, and J. Schmidhuber. A committee of neural networks for traffic sign classification. Proceedings of the IEEE International Joint Conference on Neural Networks, pages 1918-1921, 2011.

[14] L. P. Coelho. Mahotas: Open source software for scriptable computer vision. Journal of Open Research Software, 1, 2013.

[15] O. Demir and A. Y. Camurcu. Computer-aided detection of lung nodules using outer surface features. Bio-Medical Materials and Engineering, 26:1213-1222, 2015.

[16] K. Doi. Computer-aided diagnosis in medical imaging: Historical review, current status and future potential. Computerized Medical Imaging and Graphics, 31: 198-211, 2007.

[17] R. O. Duda, P. E. Hart, and D. G. Stork. Pattern Classification. 2nd edition, 2001. 
[18] B. M. Elicker and W. R. Webb. Fundamentals of High-Resolution Lung CT. Wolters Kluwer, 2013.

[19] M. Filippi, M. Rovaris, A. Campi, C. Pereira, and G. Comi. Semi-automated thresholding technique for measuring lesion volumes in multiple sclerosis: Effects of the change of the threshold on the computed lesion loads. Acta Neurologica Scandinavica, 93:30-34, 1996.

[20] O. Grove, A. E. Berglund, M. B. Schabath, H. JWL. Aerts, A. Dekker, H. Wang, E. R. Velazquez, P. Lambin, Y. Gu, and Y. Balagurunathan. Quantitative computed tomographic descriptors associate tumour shape complexity and intratumor heterogeneity with prognosis in lung adenocarcinoma. PloS one, 10(3), 2015.

[21] E. L. Hall, R. P. Kruger, S. J. Dwyer, D. L. Hall, R. W. McLaren, and G. S. Lodwick. A survey of preprocessing and feature extraction techniques for radiographic images. IEEE Transactions on Computers, 100:1032-1044, 1971.

[22] R. M. Haralick, K. Shanmugam, and H. Dinstein. Textural features for image classification. IEEE Transactions on systems, man, and cybernetics, 6:610-621, 1973.

[23] M. Javaid, M. Javid, M. Zia ur Rehman, and S. I. A. Shah. A novel approach to cad system for the detection of lung nodules in ct images. Computer Methods and Programs in Biomedicine, 135:125-139, 2016.

[24] Y. Ke and R. Sukthankar. Pca-sift: A more distinctive representation for local image descriptors. Computer Vision and Pattern Recognition, 2004.

[25] N. Kim, J. B. Seo, Y. Lee, J. G. Lee, S. S. Kim, and S. H. Kang. Development of an automatic classification system for differentiation of obstructive lung disease using hrct. Journal of Digital Imaging, 22:136-148, 2009.

[26] G. Kumar and P. K. Bhatia. A detailed review of feature extraction in image processing systems. International Conference on Advanced Computing and Communication Technologies, 2014. 
[27] D. A. Lisin, M. A. Mattar, and M. B. Blaschko. Combining local and global features for object recognition. Computer Vision and Pattern Recognition Workshops, 2005.

[28] D. G. Lowe. Object recognition from local scale-invariant features. Computer Vision, 1999.

[29] T. Messay, R. C. Hardie, and T. R. Tuinstra. Segmentation of pulmonary nodules in computed tomography using a regression neural network approach and its application to the lung image database consortium and image database resource initiative dataset. Medical Image Analysis, 22:48-62, 2015.

[30] E. N. Mortensen, H. Deng, and L. Shapiro. A sift descriptor with global context. Computer Vision and Pattern Recognition, 2005.

[31] K. Murphy, B. van Ginneken, A. M. R. Schilham, B. J. de Hoop, H. A. Gietema, and M. Prokop. A large-scale evaluation of automatic pulmonary nodule detection in chest ct using local image features and k-nearest-neighbour classification. Medical Image Analysis, 13:757-770, 2009.

[32] T. Ojala, M. Pietikainen, and D. Harwood. Performance evaluation of texture measures with classification based on kullback discrimination of distributions. Conference on Computer Vision and Image Processing, 1:582-585, 1994.

[33] H. M. Orozco, O. O. V. Villegas, V. G. C. Sanchez, H. J. O. Dominguez, and M. J. N. Alfaro. Automated system for lung nodules classification based on wavelet feature descriptor and support vector machine. Biomedical Engineering Online, 14, 2015.

[34] G. L. B. Ramalho, P. P. R. Filho, F. N. S. de Medeiros, and P. C. Cortez. Lung disease detection using feature extraction and extreme learning machine. Brazilian Journal of Biomedical Engineering, 30:207-214, 2014.

[35] P. Sermanet and Y. LeCun. Traffic sign recognition with multi-scale convolutional networks. Proceedings of the IEEE International Joint Conference on Neural Networks, pages 2809-2813, 2011. 
[36] A. A. A. Setio and et al. Validation, comparison, and combination of algorithms for automatic detection of pulmonary nodules in computed tomography images: The luna16 challenge. Medical Image Analysis, 42:1-13, 2017.

[37] R. L. Siegel, K. D. Miller, and A. Jemal. Cancer statistics, 2018. CA: A Cancer Journal for Clinicians, 68:7-30, 2018.

[38] J. Stallkamp, M. Schlipsing, J. Salmen, and C. Igel. Man vs. computer: Benchmarking machine learning algorithms for traffic sign recognition. Neural Networks, 32:323-332, 2012.

[39] S. K. TK, G. EN, and U. R. Lung nodules volume growth analysis and visualization through auto-cluster k-means segmentation and centroid/shape variance based false nodule elimination. Biomedical Research, 2017.

[40] J. Van Griethuysen, A. Fedorov, C. Parmar, A. Hosny, N. Aucoin, V. Narayan, R. Beets-Tan, J. Fillion-Robin, S. Pieper, and H. Aerts. Computational radiomics system to decode the radiographic phenotype. Cancer Research, 77:104-107, 2017.

[41] S. Wang, M. Zhou, L. Zhenyu, D. Gu, Y. Zang, D. Dong, O. Gevaert, and J. Tian. Central focused convolutional neural networks: Developing a data-driven model for lung nodule segmentation. Medical Image Analysis, 40:172-183, 2017.

[42] Y. Wang, W. Fan, P. Bhatt, and C. Davatzikos. High-dimensional pattern regression using machine learning: From medical images to continuous clinical variables. NeuroImage, 50:1519-1535, 2010.

[43] F. Zaklouta, B. Stanciulescu, and O. Hamdoun. Traffic sign classification using kd trees and random forests. Proceedings of the IEEE International Joint Conference on Neural Networks, pages 2151-2155, 2011.

[44] B. Zhao, G. Gamsu, M. S. Ginsberg, L. Jiang, and L. H. Schwartz. Automatic detection of small lung nodules on ct utilizing a local density maximum algorithm. Journal of Applied Clinical Medical Physics, 4:248-260, 2003. 
[45] S. Zhou, Y. Cheng, and S. Tamura. Automated lung segmentation and smoothing techniques for inclusion of juxtapleural nodules and pulmonary vessels on chest ct images. Biomedical Signal Processing and Control, 13:62-70, 2014.

[46] M. Zia ur Rehman, M. Javaid, S. I. A. Shah, S. O. Gilani, M. Jamil, and S. I. Butt. An appraisal of nodules detection techniques for lung cancer in ct images. Biomedical Signal Processing and Control, 41:140-151, 2018. 
Appendices 


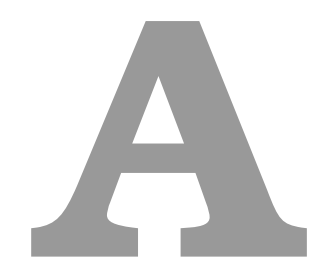

\section{Appendix A - Haralick Features and \\ Formulae}

1. Angular Second Moment

$$
f_{1}=\sum_{i} \sum_{j} p(i, j)^{2}
$$

2. Contrast

$$
f_{2}=\sum_{n=0}^{N_{g}-1} n^{2}\left\{\sum_{\substack{i=1 \\|i-j|=n}}^{N_{g}} \sum_{j=1}^{N_{g}} p(i, j)\right\}
$$

3. Correlation

$$
f_{3}=\frac{\sum_{i} \sum_{j}(i j) p(i, j)-\mu_{x} \mu_{y}}{\sigma_{x} \sigma_{y}}
$$

where $\mu_{x}, \mu_{y}, \sigma_{x}$, and $\sigma_{y}$ are the means and standard deviations of $p_{x}$ and $p_{y}$. 
4. Sum of Squares: Variance

$$
f_{4}=\sum_{i} \sum_{j}(i-\mu)^{2} p(i, j)
$$

5. Inverse Difference Moment

$$
f_{5}=\sum_{i} \sum_{j} \frac{1}{1+(i-j)^{2}} p(i, j)
$$

6. Sum Average

$$
f_{6}=\sum_{i=2}^{2 N_{g}} i p_{x+y}(i)
$$

7. Sum Variance

$$
f_{7}=\sum_{i=2}^{2 N_{g}}\left(i-f_{8}\right)^{2} p_{x+y}(i)
$$

8. Sum Entropy

$$
f_{8}=-\sum_{i=2}^{2 N_{g}} p_{x+y}(i) \log \left\{p_{x+y}(i)\right\}
$$

9. Entropy

$$
f_{9}=-\sum_{i} \sum_{j} p(i, j) \log ((i, j))
$$

10. Difference Variance

$$
f_{10}=\text { variance of } p_{x-y}
$$

11. Difference Entropy

$$
f_{11}=-\sum_{i=0}^{N_{g-1}} p_{x-y}(i) \log \left\{p_{x-y}(i)\right\}
$$

12. Information Measures of Correlation

$$
\begin{gathered}
f_{12}=\frac{H X Y-H X Y 1}{\max H X, H Y} \\
f_{13}=(1-\exp [-2.0(H X Y 2-H X Y)])^{1 / 2}
\end{gathered}
$$




$$
H X Y=-\sum_{i} \sum_{j} p(i, j) \log (p(i, j))
$$

where $H X$ and $H Y$ are entropies of $p_{x}$ and $p_{y}$, and

$$
\begin{gathered}
H X Y 1=-\sum_{i} \sum_{j} p(i, j) \log \left\{p_{x}(i) p_{y}(j)\right\} \\
H X Y 2=-\sum_{i} \sum_{j} p_{x}(i) p_{y}(j) \log \left\{p_{x}(i) p_{y}(j)\right\}
\end{gathered}
$$

13. Maximal Correlation Coeffficient

$$
f_{14}=(\text { Second largest eigenvalue of } Q)^{1 / 2}
$$

where

$$
Q(i, j)=\sum_{k} \frac{p(i, k) p(j, k)}{p_{x}(k) p_{y}(k)}
$$

